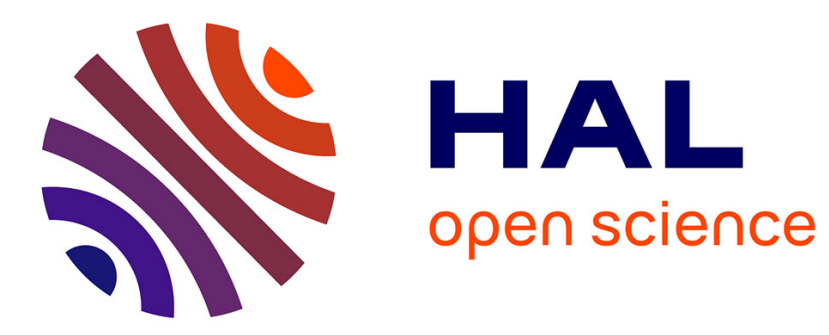

\title{
Mobiliser des indicateurs pour évaluer les effets propres des mesures agro-environnementales en France
}

\author{
Frédéric Zahm, Anne Gassiat
}

\section{To cite this version:}

Frédéric Zahm, Anne Gassiat. Mobiliser des indicateurs pour évaluer les effets propres des mesures agro-environnementales en France. Revue d'économie régionale et urbaine, 2010, 3, p. 439 - p. 471. 10.3917/reru.103.0439 . hal-00518108

\section{HAL Id: hal-00518108 \\ https://hal.science/hal-00518108}

Submitted on 16 Sep 2010

HAL is a multi-disciplinary open access archive for the deposit and dissemination of scientific research documents, whether they are published or not. The documents may come from teaching and research institutions in France or abroad, or from public or private research centers.
L'archive ouverte pluridisciplinaire HAL, est destinée au dépôt et à la diffusion de documents scientifiques de niveau recherche, publiés ou non, émanant des établissements d'enseignement et de recherche français ou étrangers, des laboratoires publics ou privés. 


\title{
Mobiliser des indicateurs pour évaluer les effets propres des mesures agro-environnementales en France.
}

\section{Agri-environmental indicators for the assessment of the treatment effects of French agri-environmental measures.}

\author{
Anne GASSIAT \\ Ingénieur chercheur \\ CEMAGREF \\ UR ADBX 50 avenue de Verdun, F-33612 Cestas Cedex, France, \\ Tel : + 33557892697 Fax : + 33557890801 \\ anne.gassiat@cemagref.fr \\ Frédéric ZAHM \\ Ingénieur chercheur \\ CEMAGREF \\ UR ADBX 50 avenue de Verdun, F-33612 Cestas Cedex, France, \\ Tel : + 33557890840 Fax : + 33557890801 \\ frederic.zahm@cemagref.fr
}

Mots-clés : évaluation ex-post, effets propres, mesures agro-environnementales, indicateurs agro-environnementaux, eau, biodiversité,

Keywords : Ex-post evaluation, Treatment effects, agri-environmental measures, agrienvironmental indicators, water, biodiversity

Classification JEL : Q18 (Agricultural policy), Q51 (evaluation of environmental effects), Q28 (Government Policy) 
Author-produced version of the article published in Revue d'Economie Régionale et Urbaine (RERU), 2010, vol. 3, p. 439 - 471 The original publication is available at http://www.cairn.info/resume.php?ID_ARTICLE=RERU_103_0439 doi : 10.3917/reru.103.0439

\section{Résumé}

L'évaluation ex-post des Mesures agro-environnementales (MAE) du Règlement de Développement Rural impose l'évaluation de leurs effets propres. La première partie de l'article rappelle les principes généraux de l'estimation des effets propres d'une politique. La démarche propose de mobiliser les indicateurs agro-environnementaux comme variables de résultats dans deux méthodes économétriques (matching et double différence). Quatre critères de sélection des indicateurs sont proposés (la pertinence de l'indicateur pour représenter la pratique agricole, le statut de l'indicateur sur la chaîne causale des effets, l'échelle spatiale de calcul, la disponibilité des variables de calcul dans les bases de données nationales) puis testés sur 220 méthodes d'indicateurs recensées. Les résultats montrent que compte tenu des variables disponibles et de la représentativité spatiale des bases de données de la statistique agricole française, seulement 40 indicateurs sont mobilisables pour évaluer les effets propres des MAE françaises sur l'eau et la biodiversité pour la période 2000 - 2006.

\section{Summary}

The ex-post evaluation of the agri-environmental Measures (AEM) of the Rural Development Programmes requires an assessment of their treatment effects. The first part of the article explains the general principles the net effects assessment procedure. The methodological framework mobilizes agrienvironmental indicators as output variables within of two econometric approaches (matching and double difference). Regarding indicators choice, four criteria for selecting indicators are first proposed (the relevance of the indicator to represent an agricultural practice, the indicators status on the causeeffect chain, the spatial scale of calculation and the availability of variables in national databases) and then tested on 220 methods based on indicators and collected from an international survey. Finally, taking into account data availability and scale representativeness of the French agricultural statistical databases, we identify 40 relevant indicators to assess the treatment effects of the French water and biodiversity AEM.

\section{- 1-}

\section{Les enjeux de l'évaluation dans la conduite de la politique étudiée}

L'évaluation des politiques publiques est un processus social et une démarche (PERRET, 2008) qui s’impose à tous les États membres de l’Union européenne ; elle participe à la vie démocratique et à l'amélioration des performances de l'action publique. La récente intégration 
Author-produced version of the article published in Revue d'Economie Régionale et Urbaine (RERU), 2010, vol. 3, p. 439 - 471 The original publication is available at http://www.cairn.info/resume.php?ID_ARTICLE=RERU_103_0439

doi : $10.3917 /$ reru. 103.0439

d’objectifs environnementaux dans la Politique Agricole Commune (PAC) implique de nouvelles démarches d'évaluation, spécifiques au traitement de la mesure des effets des programmes agro-environnementaux et en particulier des mesures agro-environnementales (MAE). Le processus d'évaluation se déroule en trois cycles : en préalable, en cours et en fin de programme (ex-ante, mi parcours, ex-post).

Cet article s'intéresse aux démarches d'évaluation ex-post en se focalisant sur l'efficacité des programmes de MAE. L'évaluation ex-post consiste à faire le point sur un programme terminé en (i) appréciant les résultats et en les comparant aux objectifs initialement fixés et aux moyens mis en œuvre (juridiques, administratifs ou financiers), (ii) s’attachant à analyser l'efficacité et l'efficience du soutien accordé. Un programme est efficient si ses effets attendus sont obtenus avec un minimum de moyens engagés. Indépendamment des moyens utilisés, l'efficacité traduit l'écart entre les objectifs initiaux et les résultats du programme et se mesure notamment à l'aide d'indicateurs.

Conformément à l'obligation d'évaluer les politiques publiques communautaires, la France a conduit en 2008, l'évaluation ex-post de son dispositif national de MAE sur la période 2000 - 2006 en s’attachant à respecter les lignes directrices et les questions évaluatives communes imposées par la COMMISSION EUROPEENNE (2000). Cette évaluation a comme principal objectif l'analyse des « effets propres » ${ }^{1}$ des MAE (Commission européenne, 1999a). Si le cadre méthodologique communautaire impose les indicateurs comme outils de suivi et d'analyse (COMMISsion EUROPEENNE, 2000), nous proposons d'aller au-delà de la pratique courante, qui est d'utiliser les taux de réalisation comme indicateurs d'efficacité d'une politique (GUERIN et RUAS, 2006), en tentant d'apprécier l'effet propre à partir d'une démarche intégratrice associant indicateurs et méthodes économétriques. Pour LOFREDI et al. (2005), l'évaluation des effets propres d'un programme se heurte à deux problèmes : (1) le choix et la disponibilité d'indicateurs de résultats ou de performance des MAE et (2) l'impossibilité de comparer directement les performances environnementales des agriculteurs après la mise en place de la mesure (la MAE dans notre cas) avec la situation contrefactuelle (c'est-à-dire ce qu'auraient été les performances environnementales des agriculteurs en absence de la mesure). Dans cet article, nous porterons notre attention sur les indicateurs en

\footnotetext{
${ }^{1}$ cf. définition en section 2
} 
Author-produced version of the article published in Revue d'Economie Régionale et Urbaine (RERU), 2010, vol. 3, p. 439 - 471 The original publication is available at http://www.cairn.info/resume.php?ID_ARTICLE=RERU_103_0439

doi : $10.3917 /$ reru. 103.0439

tenant compte des spécificités imposées par les méthodes économétriques pour résoudre ce second problème lié à la mesure du contrefactuel.

Bien que le cadre commun d’évaluation soit imposé, la Commission européenne laisse l’initiative à chaque État membre de développer des méthodes scientifiques et de déterminer les indicateurs agro-environnementaux pertinents pour estimer les effets propres des MAE. Cette situation pourrait paraître surprenante étant donnée l’importance financière des dépenses publiques allouées à ces mesures (en France : 3,4 milliards d'euros, soit 33 \% des dépenses publiques totales du développement rural sur la période 2000-2006). Trois raisons permettent d'expliquer cette situation :

- étant donné «la jeunesse relative» des politiques agro-environnementales, l'évaluateur est encore aujourd'hui confronté à un manque de méthodes adaptées pour l'évaluation des effets propres dans le domaine de l'agro-environnement (GUERIN, 2008 ; ZAHM et al., 2008a). Mobilisées depuis une quinzaine d'année pour l'évaluation des politiques publiques de l'économie du travail (BeHAghel et al., 2009 ; CREPON et DesPlatz, 2001 ; Even et Klein, 2007) et de l'éducation (Benabou et al., 2004 ; 2009), elles restent délicates dans leur protocole de mise en œuvre ;

- la possibilité de mobiliser les indicateurs dans des approches quantitatives est encore délicate. Les données pour leur calcul restent souvent un obstacle pour des évaluations à l'échelle nationale. ZALIDIS et al. (2004) notent que malgré le travail considérable réalisé ces dernières années pour identifier des indicateurs agro-environnementaux appropriés, ce travail de sélection final n’a pas encore été conduit jusqu’à son terme ;

- l’évaluateur n'évalue pas la politique communautaire mais son application nationale. Aussi, le nombre important de MAE, la complexité et les nombreuses évolutions réglementaires des dispositifs nationaux n’ont pas contribué à la stabilisation d'une méthode européenne.

L’objectif de cet article est (i) de rappeler les principes généraux de l'estimation des effets propres des MAE à partir d'une méthode couplant indicateurs agro-environnementaux et méthodes économétriques puis (ii) d'analyser pour le dispositif agro-environnemental français quels sont les indicateurs susceptibles d'être mobilisés dans les méthodes économétriques. Les résultats porteront sur les indicateurs agro-environnementaux mobilisables pour évaluer les MAE ayant pour objectif la préservation de l'eau (qualité et quantité) et de la biodiversité 
Author-produced version of the article published in Revue d'Economie Régionale et Urbaine (RERU), 2010, vol. 3, p. 439 - 471 The original publication is available at http://www.cairn.info/resume.php?ID_ARTICLE=RERU_103_0439

doi : $10.3917 /$ reru.103.0439

(biodiversité naturelle ou cultivée sur l'exploitation). Nous avons porté notre attention sur ces deux enjeux car ils sont aujourd'hui prioritaires dans le Plan Stratégique National² (MINISTERE DE L’AGRICULTURE ET DE LA PECHE, 2006) et les mesures les concernant ont été parmi les plus souscrites au cours de la période 2000-2006.

Après avoir rappelé le cadre général de l'évaluation des effets propres de la politique étudiée et de l'utilisation d'indicateurs agro-environnementaux (section 2), nous présentons notre cadre méthodologique pour sélectionner des indicateurs agro-environnementaux adaptés à notre approche (section 3) et l'appliquons (section 4) au dispositif des MAE françaises sur la période 2000-2006. Nous concluons à la fois sur des recommandations pour la prochaine évaluation de la programmation agro-environnementale 2007-2013 ainsi que sur des éléments d'agenda pour un programme de recherche.

\section{Évaluer une politique : les effets propres et les indicateurs}

Le cadre méthodologique proposé pour l'évaluation des effets propres des MAE s'articule en deux étapes: d'abord sélectionner des indicateurs mesurant l'impact environnemental attendu des pratiques agricoles ( $1^{\text {ère }}$ étape) puis déterminer l'effet propre de la MAE sur ces indicateurs en mobilisant les travaux issus de l'économétrie des effets de traitement ( $2^{\text {nde }}$ étape).

Après un rappel des principes généraux de la seconde étape, présentant les méthodes économétriques susceptibles d'évaluer des effets propres des MAE, nous consacrons la suite de notre article à la première étape sur les indicateurs agro-environnementaux.

\section{1. Évaluer les effets propres d'une politique agro-environnementale...}

L'effet propre d'une politique se définit comme la différence entre la situation en présence de la politique et la situation qui aurait prévalu en son absence. Dans le cas d'évaluations ex-post, la situation en présence de la politique est observée. Il faut alors déterminer ce qu'il serait advenu en l'absence de la politique. Cette dernière situation,

\footnotetext{
${ }^{2}$ PSN : analyse économique, sociale et environnementale des zones rurales permettant d'identifier les principaux enjeux de la stratégie nationale de développement rural au regard des orientations stratégiques communautaires et dans un souci de valoriser durablement les territoires ruraux.
} 
Author-produced version of the article published in Revue d'Economie Régionale et Urbaine (RERU), 2010, vol. 3, p. 439 - 471 The original publication is available at http://www.cairn.info/resume.php?ID_ARTICLE=RERU_103_0439

doi : $10.3917 /$ reru. 103.0439

hypothétique et inobservée, est appelée «situation contrefactuelle », « contrefactuel » ou « antimonde ».

Au plan théorique, la résolution économique de ce problème du contrefactuel mobilise le modèle causal conçu initialement par RUBIN (1974) pour l'analyse des effets des traitements médicaux (BRODATY et al., 2002 ; SCHMITT et al., 2003). Ce modèle canonique a d'abord été transposé pour l'évaluation des politiques de l'emploi sous l'impulsion de travaux d'économètres tels que HECKMAN, LALONDE et SMITH (1999) qui ont été au centre d'une littérature économétrique riche en avancées méthodologiques depuis les années 1990 (BRODATY et al., 2002).

Appliqué aux MAE, ce modèle théorique revient à estimer leur contribution spécifique au maintien, à la protection ou à l'amélioration des ressources naturelles (qualité du sol, qualité et quantité de l'eau), de la biodiversité (faune et de la flore, habitats, races d'animaux ou variétés végétales) et du paysage. Mais, la simple observation des changements de pratiques chez les agriculteurs bénéficiaires de MAE n'est pas un critère suffisant pour juger de l'effet d'une mesure. Il importe en effet de pouvoir déterminer la part des changements de pratiques observés qui résulte effectivement de la contractualisation de MAE.

$\mathrm{Au}$ plan méthodologique, cette question implique de déterminer la situation contrefactuelle à partir des données observées, c'est-à-dire d'apprécier ce qui se serait passé si le programme de MAE n'avait pas eu lieu. Cette démarche revient à rendre compte des effets propres des MAE. Cet effet propre se définit comme la différence entre les pratiques agricoles observées en présence de la politique (bénéficiaires) et celles que l’on aurait observées en l'absence de la politique dans un groupe d'agriculteurs qualifiés de contrefactuels (c'est-à-dire d'agriculteurs non bénéficiaires mais présentant les mêmes caractéristiques que les bénéficiaires). Naturellement, il n’est pas possible d’observer simultanément ces deux situations. Par conséquent, l'évaluation de l'effet propre d'une politique agroenvironnementale est le fruit d'une reconstitution intellectuelle (GIBERT, 2003) car elle revient à comparer une situation observée chez les agriculteurs bénéficiaires de la MAE à la fin du programme à la situation qui aurait été observée si le programme de MAE n’avait pas été mis en œuvre. CHABE-FERRET et SuBERVIE (2008) rappellent que l'objectif est de parvenir à un estimateur sans biais de l'effet moyen des mesures sur les bénéficiaires et de l'effet moyen conditionnel à certaines caractéristiques des bénéficiaires. Pour recréer la situation 
Author-produced version of the article published in Revue d'Economie Régionale et Urbaine (RERU), 2010, vol. 3, p. 439 - 471 The original publication is available at http://www.cairn.info/resume.php?ID_ARTICLE=RERU_103_0439

doi : $10.3917 /$ reru. 103.0439

contrefactuelle à partir des données observées, MAGNAC (2000) identifie trois grandes familles de méthodes :

- Les méthodes expérimentales, basées sur une participation aléatoire des bénéficiaires à la politique, qui garantissent l'absence de biais de sélection. Ce sont les méthodes les plus robustes, et pour lesquelles les outils statistiques mobilisés pour l'analyse des résultats sont les plus simples (notamment comparaison de moyennes avec/sans). Ces méthodes expérimentales ont récemment été utilisées en France pour conduire l'évaluation du Revenu de Solidarité Active (Bourguignon, 2009) et des politiques actives de retour vers l'emploi (BEHAGHEL et al., 2009).

- Les méthodes structurelles, qui peuvent être utilisées indifféremment pour une évaluation ex-ante ou ex-post. Elles reconstruisent la situation contrefactuelle à l'aide de simulations d'un modèle décrivant le comportement des agents, dont les paramètres ont été estimés ou calibrés.

- Les méthodes économétriques corrigeant le biais de sélection en cas d'une participation non aléatoire à la politique, ce qui est notre cas pour les politiques agroenvironnementales. Ces méthodes permettent d'estimer l'effet propre de la politique à partir des données observées. On distingue les méthodes basées sur l’hypothèse que le processus de participation des agriculteurs dans la politique peut être saisi au travers de variables observées et les méthodes permettant de relâcher cette hypothèse (CHABE-FERRET et SUBERVIE, 2008).

Magnac (2000) conclut que la transposition des méthodes économétriques aux évaluations des politiques de développement rural est possible. Toutefois, PRIMDAHL et al. (2003) soulignent que de nombreuses difficultés méthodologiques persistent, malgré les efforts conduits notamment par l’OCDE (1999b) ces dernières années. L’analyse empirique de GUERIN et RUAS (2006) sur 72 évaluations ex-post réalisées de 1990 à 2003 confirme que ces méthodes contrefactuelles n’ont jamais été véritablement employées dans le champ des politiques du développement rural à l'exception de la récente avancée méthodologique appliquée à l'estimation des effets propres de programmes de développement de zones rurales (LOFREDi, 2007 ; LOFREdi et SCHMiTT, 2005). Chabe-FerRet et SuberVIE (2008) complètent ce constat et montrent que les démarches d'évaluation mises en œuvre dans l'évaluation des MAE aujourd'hui présentent deux types de biais. Il s’agit (i) du biais de sélection lorsque les méthodes employées comparent le niveau moyen des pratiques agricoles d'agriculteurs bénéficiaires et de non bénéficiaires (méthode dites avec / sans) ou (ii) du biais de tendance 
Author-produced version of the article published in Revue d'Economie Régionale et Urbaine (RERU), 2010, vol. 3, p. 439 - 471 The original publication is available at http://www.cairn.info/resume.php?ID_ARTICLE=RERU_103_0439

doi : $10.3917 /$ reru. 103.0439

temporelle lorsque les méthodes comparent le niveau moyen de pratiques des bénéficiaires après la contractualisation au niveau moyen de leurs pratiques avant la contractualisation (méthode qualifiée de avant / après).

C'est pour lever ces biais que CHABE-FERRET et SubERVIE (2008) proposent d'utiliser des méthodes statistiques, actuellement innovantes en agro-environnement (méthodes du matching et de la double différence) mais déjà mobilisées notamment par CREPON et DESPLATZ (2001) pour estimer l'effet net de la réduction des charges sociales sur la création d'emplois salariés en France. Ces différentes méthodes mobilisent une ou des variables objectives susceptibles d'être affectées par la politique en question. C'est la différence entre le niveau de cette variable en présence de la politique et son niveau en l'absence de la politique qui constitue l'effet de la politique.

Pour l'évaluation des MAE, ces variables sont des indicateurs agro-environnementaux (cf. définition au point 2.2). Dans ce cas, l'effet propre se mesure comme la différence entre le niveau de l'indicateur observé en présence de la politique et le niveau de l'indicateur que l'on aurait observé en l’absence de la politique (appelé contrefactuel). Cette différence mesure alors bien ce qui résulte effectivement de la politique.

Figure 1: $\quad$ Illustration de l'effet propre pour l'indicateur quantité d’azote.

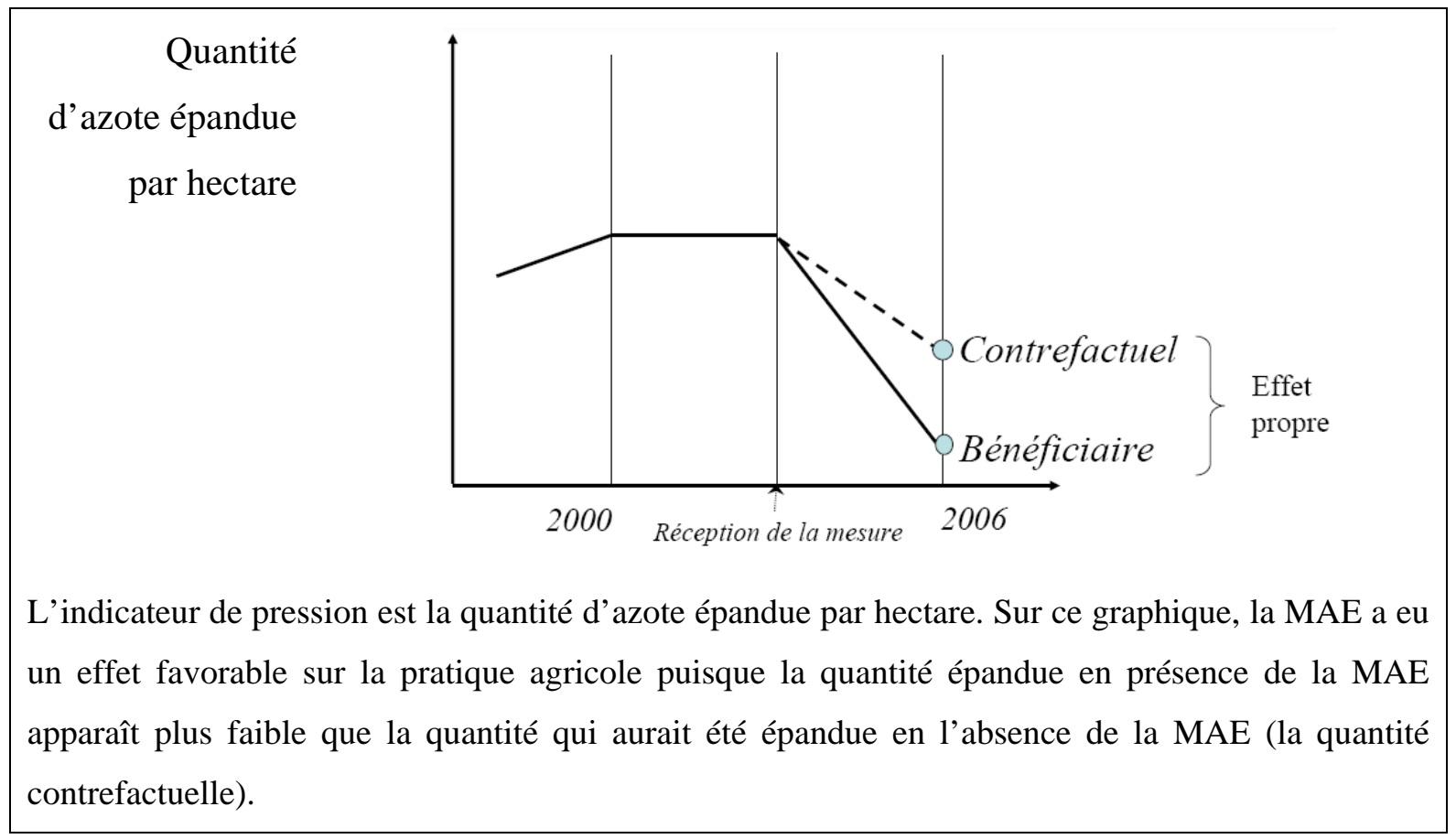

Source : CHABE-FERRET et SubERVIE, 2008 
Author-produced version of the article published in Revue d'Economie Régionale et Urbaine (RERU), 2010, vol. 3, p. 439 - 471 The original publication is available at http://www.cairn.info/resume.php?ID_ARTICLE=RERU_103_0439 doi : $10.3917 /$ reru. 103.0439

\section{2. ... à partir d'indicateurs}

MAURIZI et VerRel (2002) rappellent qu'il n'existe pas de définition universelle d'un indicateur car chaque institution ou auteur recourt à sa propre définition qui est fonction des objectifs et du public concerné. Un indicateur est « une grandeur qui fournit une information au sujet d'une variable plus difficile d'accès ou d'un système plus complexe afin d'aider un utilisateur dans son action (prise de décision, construction d'un programme d'action, modélisation....) » (BOCKSTALLER et GIRARDIN, 2003 ; GrAS et al., 1989). La finalité d'un indicateur est de réduire l'information pour rendre cette réalité accessible aux utilisateurs ou décideurs sous forme d'outil de diagnostic ou d'aide à la décision (GIRARDIN et al., 1999).

Pour Mitchell et al. (1995), les indicateurs sont des mesures « alternatives qui sont utilisées pour décrire une situation quand il n’est pas possible, pour des raisons techniques ou financières, de l'évaluer directement ». Théoriquement le recours à la modélisation pour estimer des variables pourrait se substituer à des mesures sur le terrain pour pallier les difficultés de collectes. Cependant, le manque actuel de modèles opérationnels utilisant des données facilement disponibles reste encore un obstacle majeur à l'estimation de ces mesures (BOCKSTALLER et al., 1997).

Les indicateurs sont donc des outils permettant la mesure simple, compréhensible, la plupart du temps indirecte de la situation par rapport à un objectif donné. En agriculture, pour évaluer la performance environnementale des changements de pratiques agricoles, c'est la notion d'indicateur agro-environnemental qui a été retenue par l'OCDE dans son modèle causal « Pression - State - Response » (PSR) (OCDE, 1999 a et b). Ce modèle repose sur le fait que les activités humaines exercent des pressions (P) sur l'environnement, modifient son état ( $\mathrm{S}$ ) et la société y répond (R) en adaptant ses comportements ou politiques. L’OCDE (2001) distingue trois types d'indicateurs agro-environnementaux dans cette chaine causale des effets :

- les indicateurs de pression (P) visent à mettre en évidence l'effet environnemental des pratiques agricoles sur l'environnement. Ce concept de pression fait référence aux causes qui engendrent des changements d'état et des impacts. La pression peut être estimée directement par les émissions de polluants ou bien indirectement par les types d'activités agricoles. 
Author-produced version of the article published in Revue d'Economie Régionale et Urbaine (RERU), 2010, vol. 3, p. 439 - 471 The original publication is available at http://www.cairn.info/resume.php?ID_ARTICLE=RERU_103_0439 doi : $10.3917 /$ reru. 103.0439

- les indicateurs d'état (S) correspondent à des mesures directes de l'état du milieu (flore présente, concentration d’une matière active dans l'eau, etc..). Leur interprétation permet d'élaborer un diagnostic.

- les indicateurs de réponse ou de suivi (R) évaluent dans quelles mesures les modifications de pratiques via entre autres des programmes d'action ont atteint les objectifs fixés.

Ce statut de l'indicateur dans la chaîne causale des effets est un critère de choix déterminant (BocKstaller et al., 2008). Au plan théorique, NiEMEIJER et De Groot (2008) soulignent la nécessité de positionner les indicateurs dans la chaîne causale des effets des deux cadres conceptuels les plus couramment utilisés : le modèle PSR pour l’OCDE (1999a et b) et le modèle DPSIR (Driving Force - Pression - State - Impact - Response) pour la Commission européenne (EEA, 1999). Le modèle DPSIR est une adaptation du modèle PSR avec deux indicateurs supplémentaires: (1) les indicateurs de force motrice (D) décrivent le développement social, économique et démographique de la société et leur implication dans les changements de consommation ou de production, (2) les indicateurs d'impact (I) caractérisent les effets ultimes causés par les changements d'état. Nous proposons de combiner ces deux modèles et au final de retenir 4 types d'indicateurs : état, suivi, pression et impact.

S'agissant des indicateurs d'état, ils sont peu disponibles, en particulier dans le domaine de la biodiversité, car peu de bases de données nationales leurs sont dédiées et leur collecte reste relativement dispersée. Pour l'évaluation de la qualité de l'eau, si les mesures directes semblent être le moyen le plus évident pour évaluer l'effet des pratiques agricoles, elles ne sont pas généralisables pour une question de coût d'intervention sur le terrain et de coût d'analyse. GiRARDiN et BocKSTALLER (1997) notent que l'interprétation de ces indicateurs d'état pose des problèmes en raison de l'échantillonnage et de la variabilité des conditions du milieu au cours du temps. Par ailleurs, il est difficile (voire impossible) de disposer d'indicateurs susceptibles d'allouer la contribution individuelle d’un bénéficiaire à la modification de l'état global du territoire ou d’un écosystème. En effet, les processus de pollution diffuse liés aux intrants (pesticides, azote) mettent en jeu des mécanismes de transferts à la fois complexes et multifactoriels qui posent la question des effets non linéaires liés au changement d’échelle.

Quant aux indicateurs de suivi, nous proposons de ne pas les retenir car nous rejoignons GUERIN et RUAS (2006) qui notent qu'« une politique peut certes être efficace du 
Author-produced version of the article published in Revue d'Economie Régionale et Urbaine (RERU), 2010, vol. 3, p. 439 - 471 The original publication is available at http://www.cairn.info/resume.php?ID_ARTICLE=RERU_103_0439

doi : $10.3917 /$ reru. 103.0439

point de vue des réalisations, mais inefficace sous l'angle de ses impacts ». Ainsi les mesures « réduction des intrants » ont été largement contractualisées alors que leurs impacts sur l'environnement étaient très faibles compte tenu d'une absence de localisation ciblée obligatoire et d'un cahier des charges peu contraignant.

Les indicateurs d'impact sont en théorie ceux qu'il conviendrait de retenir car compte tenu de leur position sur la chaine causale des effets, ils sont les plus à même de rendre compte de l'effet des pratiques sur le milieu naturel (ZAHм et al., 2008b). Au plan de leur construction scientifique Udo DE HAEs et al. (1999) distinguent deux types d'indicateurs selon le niveau de l'agrégation :

- d'une part, les indicateurs « midpoint », pour lesquels la technique d’agrégation est construite à l'émission. Toutes les substances polluantes émises contribuant à un même impact sont alors agrégées pour donner un indicateur (ex. : l'émission de chacun des gaz à effet de serre est calculée en équivalents CO2 puis est sommée en un indicateur changement climatique) ;

- d'autre part les indicateurs « endpoint » dont l'agrégation de l'effet final est construite par une modélisation d’impacts finaux observables.

Notre analyse des travaux sur la construction de ces indicateurs d'impact (CLERGUE et al., 2005 ; DeVILlers J. et al, 2005 ; LeVReL H., 2006) montre qu'ils mobilisent, pour leur calcul, des variables souvent très difficiles à obtenir et donc pratiquement jamais disponibles dans les bases de données publiques (par exemple distances des parcelles au cours d'eau, hauteur de la plante à la date du traitement, densité du sol, richesse spécifique de la flore prairiale, etc.)

C’est pourquoi aujourd'hui, lorsqu'on souhaite évaluer l'effet des changements de pratiques agricoles, les travaux de PAYRAUDEAU et VAN der WERF (2005) complétés par LÜTZ et FELICI (2009) montrent que ce sont les indicateurs de pression, qui sont le plus à même de rendre compte de l'effet potentiel des pratiques agricoles. Pour l'enjeu qualité de l'eau, l'intérêt de ce type d'indicateur est lié à la spécificité des problématiques de pollution diffuse, car l'effet d'un changement de pratique ou d'activité agricole sur la variable d'état n'est souvent pas mesurable ou observable directement chez les bénéficiaires de la mesure. En se basant sur leur position dans la chaîne causale des effets reliant les pratiques de l'agriculteur aux impacts environnementaux, BocKSTALLER et al. (2008) classent les indicateurs de 
Author-produced version of the article published in Revue d'Economie Régionale et Urbaine (RERU), 2010, vol. 3, p. 439 - 471 The original publication is available at http://www.cairn.info/resume.php?ID_ARTICLE=RERU_103_0439

doi : $10.3917 /$ reru. 103.0439

pression en deux catégories : (1) les indicateurs basés sur les pratiques de l'agriculteur : ils peuvent être qualitatifs (mise en œuvre de telle ou telle technique) ou bien quantitatifs (utilisation de tel intrant à telle dose), (2) les indicateurs estimant les émissions de polluants (NO3, CO2, pesticides, ...) ; ces indicateurs peuvent être basés sur des approches très simples, par exemple des bilans intrants - sortants (pour l'azote, le phosphore, les métaux lourds, ...) ou bien sur des modèles de simulation plus ou moins sophistiqués.

En définitive, nous retiendrons qu'un indicateur agro-environnemental de pression contribue à apporter une appréhension sur l'effet environnemental par une évaluation indirecte des changements de pratiques. Dans la démarche que nous proposons, le statut de l'indicateur n’est pas le seul critère de sélection d'indicateurs mobilisables pour estimer les effets propres des MAE. Nous montrons dans la partie suivante qu’il doit être associé à trois autres critères.

- 3-

\section{Méthode de sélection des indicateurs : critères et disponibilité}

Au-delà du statut de l'indicateur, il convient de préciser (1) les autres critères de sélection des indicateurs agro-environnementaux et (2) les bases de données mobilisables pour leur calcul, afin que ces derniers puissent être intégrés dans les méthodes économétriques des effets de traitements.

\subsection{Quatre critères pour choisir les indicateurs pertinents}

Les indicateurs pertinents doivent d'abord répondre à un certain nombre de critères communément admis : être analytiquement et scientifiquement valides, pertinents par rapport à la mesure étudiée et sensibles aux changements (DONNELly et al., 2007 ; BocKSTALLER et Girardin, 2003 ; NiemeiJer et De Groot, 2008 ; Piorr, 2003 ; Schomaker, 1997). Nous proposons de mobiliser ensuite quatre autres critères présentés en détail ci-dessous, si l'on souhaite les utiliser dans une méthode d'estimation des effets propres. 
Author-produced version of the article published in Revue d'Economie Régionale et Urbaine (RERU), 2010, vol. 3, p. 439 - 471 The original publication is available at http://www.cairn.info/resume.php?ID_ARTICLE=RERU_103_0439 doi : 10.3917/reru.103.0439

\section{Critère 1 : le lien entre l'indicateur, l'enjeu environnemental et les pratiques agricoles}

Il s'agit d'identifier le ou les indicateurs pertinents au regard de l'enjeu environnemental et de la pratique agricole associée à la MAE. Nous nous intéressons aux trois principaux enjeux environnementaux proposés dans le cadre du dispositif MAE : ressources naturelles (qualité des sols, qualité et quantité de l'eau), biodiversité et paysage. Dans un premier temps, à partir du travail typologique croisant enjeux et pratiques agricoles, réalisé lors de l'évaluation à mi-parcours des MAE en 2003 (OREADE-BRECHE, 2005), ces trois enjeux sont déclinés en 18 sous-enjeux codés (de SOL1 à BIOr3) et classées selon les questions évaluatives de la Commission européenne (cf. annexe 1). Puis chaque indicateur est relié à un sous-enjeu et à la ou les pratique(s) agricole(s) qu'il est susceptible d'évaluer. Enfin, il est proposé une note sur une échelle de 1 à 3 (liens faible, moyen, fort) qui rend compte de la capacité de l'indicateur à évaluer la ou les pratiques agricoles. Cette note qualitative est attribuée, soit en fonction des informations contenues dans les publications qui se réfèrent à l'indicateur, soit à défaut en mobilisant notre propre expertise agronomique.

\section{Critère 2: le statut de l'indicateur sur la chaîne causale des effets}

Les indicateurs sont classés en fonction des quatre statuts présentés en section 2 : état, suivi, pression et impact

Tableau 1: Statut des indicateurs agro-environnementaux

\begin{tabular}{|c|c|c|c|c|}
\hline \multirow{2}{*}{$\begin{array}{c}\text { Statut de } \\
\text { l'indicateur }\end{array}$} & \multirow{2}{*}{ Description } & \multirow{2}{*}{ Objectifs } & \multicolumn{2}{|c|}{ Exemple } \\
\hline & & & Indicateurs & Objectifs \\
\hline $\begin{array}{l}\text { Indicateurs de } \\
\text { pression } \\
\text { (P) }\end{array}$ & $\begin{array}{l}\text { Décrire les effets } \\
\text { potentiels des } \\
\text { activités agricoles sur } \\
\text { l'environnement }\end{array}$ & $\begin{array}{l}\text { Qualifier et quantifier les } \\
\text { relations et conditions agro- } \\
\text { environnementales sous- } \\
\text { jacentes entre les pratiques } \\
\text { agricoles et l'état de } \\
\text { l'environnement }\end{array}$ & $\begin{array}{l}\text { Solde du bilan } \\
\text { apparent de l'azote }\end{array}$ & $\begin{array}{l}\text { Rendre compte } \\
\text { des effets des } \\
\text { MAE sur la } \\
\text { qualité de l'eau } \\
\text { suite à une } \\
\text { modification de } \\
\text { la fertilisation }\end{array}$ \\
\hline $\begin{array}{l}\text { Indicateurs } \\
\text { d'état } \\
\text { (S) }\end{array}$ & $\begin{array}{l}\text { Renseigner sur l'état } \\
\text { des milieux naturels } \\
\text { (à partir de données } \\
\text { bio-physico- } \\
\text { chimiques) }\end{array}$ & $\begin{array}{l}\text { Établir un diagnostic ou une } \\
\text { situation de référence }\end{array}$ & $\begin{array}{l}\text { Taux d'azote dans } \\
\text { l'eau } \\
\text { Niveau des nappes } \\
\text { phréatiques }\end{array}$ & $\begin{array}{l}\text { Rendre compte } \\
\text { de la qualité } \\
\text { intrinsèque (ou } \\
\text { quantité) du } \\
\text { milieu aquatique } \\
\end{array}$ \\
\hline $\begin{array}{l}\text { Indicateurs } \\
\text { d'impact } \\
\text { (I) }\end{array}$ & $\begin{array}{l}\text { Décrire les effets } \\
\text { ultimes causés par les } \\
\text { changements de } \\
\text { pratiques (agricoles) }\end{array}$ & $\begin{array}{l}\text { Mesurer la pression, ainsi } \\
\text { que le risque associé à la } \\
\text { pression (croisement de la } \\
\text { pression et de la sensibilité } \\
\text { environnementale) }\end{array}$ & $\begin{array}{l}\text { Taux de mortalité } \\
\text { des individus, } \\
\text { Espérance de vie }\end{array}$ & $\begin{array}{l}\text { Rendre compte } \\
\text { de l'impact du } \\
\text { programme au } \\
\text { niveau global et } \\
\text { spécifique }\end{array}$ \\
\hline
\end{tabular}


Author-produced version of the article published in Revue d'Economie Régionale et Urbaine (RERU), 2010, vol. 3, p. 439 - 471 The original publication is available at http://www.cairn.info/resume.php?ID_ARTICLE=RERU_103_0439 doi : 10.3917/reru.103.0439

Statut de

l'indicateur

\begin{tabular}{|c|c|}
\hline $\begin{array}{l}\text { Indicateurs de } \\
\text { suivi } \\
\text { (R) }\end{array}$ & $\begin{array}{l}\text { Informer sur la } \\
\text { consommation des } \\
\text { ressources } \\
\text { financières et la } \\
\text { situation des } \\
\text { réalisations }\end{array}$ \\
\hline
\end{tabular}

Description
Objectifs

Élaborer le système de suivi
du programme

Exemple

Indicateurs

Objectifs

Nombre de

bénéficiaires

Taux de surfaces contractualisées
Rendre compte des réalisations du programme

\section{Critère 3 : l'échelle spatiale de calcul}

Comme précisé précédemment, l’identification des effets propres des MAE implique de mobiliser des groupes d'agriculteurs bénéficiaires et de construire leurs contrefactuels. Cette démarche impose de mobiliser essentiellement des données à l'échelle de l'exploitation, pour pouvoir comparer ces deux groupes d'exploitants. Or un indicateur est toujours construit en fonction d'une échelle spatiale en principe précisée dans sa méthode initiale, il peut donc perdre de son sens à une autre échelle de calcul. Aussi, nous avons codé les indicateurs en fonction des quatre échelles spatiales : parcelle agricole, exploitation, zonages à enjeu environnemental, zonages administratifs (commune, département, région, nation).

\section{Critère 4 : les variables de calcul et leur disponibilité dans les bases de données nationales}

Le quatrième et dernier critère porte sur la possibilité de calculer les indicateurs. Les données utilisées en évaluation de politique agro-environnementale font appel à :

- des données dites secondaires, qui sont déjà collectées à partir d'enquêtes officielles (statistique agricole, ...) ou bien renseignées dans des dossiers administratifs (demandes d'aides agricoles, ...) voire lors des évaluations à miparcours,

- des données dites primaires, spécifiquement recueillies par l’évaluateur, le plus souvent auprès des bénéficiaires (questionnaires, entretiens ou études de cas constituent alors les principaux outils d'enquête).

Ce quatrième critère nous permet d'écarter volontairement tous les indicateurs qui mobiliseraient des données primaires. En effet, le couplage proposé dans la méthode d'évaluation des effets propres a comme objectif final d'être opérationnel sur toute la France et d'être transposable aux autres États membres, ce qui nous amène également à étudier les bases de données disponibles et mobilisables. 
Author-produced version of the article published in Revue d'Economie Régionale et Urbaine (RERU), 2010, vol. 3, p. 439 - 471 The original publication is available at http://www.cairn.info/resume.php?ID_ARTICLE=RERU_103_0439 doi : 10.3917/reru.103.0439

\subsection{Quelles bases de données mobiliser?}

Les bases de données à mobiliser doivent disposer à la fois des variables nécessaires au calcul de chaque indicateur mais aussi des informations minimales pour l'application des méthodes économétriques. Les méthodes économétriques mobilisent des traitements statistiques qui imposent de disposer (i) d'un nombre important d'exploitations, (ii) d'informations sur les caractéristiques des exploitants et des exploitations et (iii) d’informations sur les pratiques agricoles des exploitants bénéficiaires ou non.

Notre analyse révèle qu'au moins 5 bases de données sont disponibles pour appliquer la démarche d'évaluation des effets propres. Il s’agit :

- des quatre bases statistiques du $\mathrm{SSP}^{3}$ (DATAGRESTE). Le Recensement agricole (RA), réalisé en 2000, constitue la base statistique de toutes les exploitations agricoles. Tous les deux ans, l'enquête sur les structures des exploitations agricoles (STRUCTURE) permet d'actualiser à partir d'un échantillon d'enquêtés la situation des exploitations à l'échelle du département. L’enquête sur les pratiques culturales (PK), qui a lieu tous les cinq ans à l'échelle de la parcelle, fournit des informations sur les itinéraires techniques des principales cultures. Enfin, le réseau d’informations comptables agricoles (RICA) collecte annuellement les principales variables économiques susceptibles d’apprécier les coûts de production agricole.

Tableau 2: $\quad$ Les bases de données du SSP

\begin{tabular}{|c|c|c|c|c|}
\hline & $\mathbf{R A}$ & STRUCTURE & PK & RICA \\
\hline Périodicité & 10 ans & 2 ans & 2001, 2006 & Chaque année \\
\hline Échantillon & $\begin{array}{c}664000 \\
\text { exploitations }\end{array}$ & $\begin{array}{c}76500 \\
\text { exploitations }\end{array}$ & $\begin{array}{c}18500 \\
\text { parcelles }\end{array}$ & $\begin{array}{c}\quad 7300 \\
\text { exploitations }\end{array}$ \\
\hline $\begin{array}{l}\text { Échelle de } \\
\text { représentativité }\end{array}$ & Communes & Départements & $\begin{array}{l}\text { Région + zones } \\
\text { vulnérables }\end{array}$ & $\begin{array}{l}\text { Région (toutes OTEX } \\
\text { confondues) } \\
\text { France (par OTEX) }\end{array}$ \\
\hline
\end{tabular}

OTEX: orientations technico-économiques des exploitations agricoles

- de la base administrative de données OSIRIS de l'Agence Spéciale de Paiement (ASP). Elle fournit les données individuelles sur les exploitations ayant contracté une MAE. Si

\footnotetext{
${ }^{3}$ SSP: Service de la Statistique et de la Prospective du ministère de l'Alimentation, de l'Agriculture et de la Pêche
} 
Author-produced version of the article published in Revue d'Economie Régionale et Urbaine (RERU), 2010, vol. 3, p. 439 - 471 The original publication is available at http://www.cairn.info/resume.php?ID_ARTICLE=RERU_103_0439

doi : $10.3917 /$ reru. 103.0439

ses fichiers comportent les données de réalisation propres à chaque dispositif (code de la mesure, montant payé, surface contractualisée, date de versement, etc..), ils comprennent très peu d’informations sur les caractéristiques structurelles de chaque bénéficiaire.

L’Observatoire du Développement Rural $(\mathrm{ODR})^{4}$ a été développé en 2006, par l'Institut National de Recherche Agronomique (INRA) à la demande du ministère de l'Alimentation, de l'Agriculture et de la Pêche (MAAP) pour notamment contribuer à la structuration des données préparatoires à l'évaluation. Il comporte des informations sur le suivi spatial et quantitatif des réalisations des MAE (données de l'ASP), ainsi que des données statistiques (issues essentiellement de DATAGRESTE) dans la mesure du possible à l'échelle communale.

L’analyse de ces bases de données montre que leur capacité à rendre compte de résultats à différentes échelles géographiques est relativement faible. Seuls le RA et les bases de données sur les bénéficiaires de MAE disposent de l'exhaustivité des exploitations agricoles et donc d'une représentativité à l'échelle communale, les autres enquêtes portent toutes sur des échantillons et leur représentativité ne va donc pas en deçà du département.

\section{- 4-}

\section{Application au domaine des MAE : l'existant et le souhaitable}

Trois grandes étapes marquent l’intégration de la dimension environnementale dans la PAC (BonnieuX, 2009). A partir de 1985, le règlement européen CE/797/85 (article 19) autorise pour la première fois le versement d'aides nationales aux exploitations agricoles situées en zones sensibles "ayant un intérêt reconnu du point de vue de l'écologie et du paysage". Cet objectif environnemental se renforce dès 1992, avec la réforme de la PAC (CE/2078/92), qui instaure des mesures agro-environnementales (MAE) avec un cofinancement par l'Union européenne. Ces dernières deviennent alors d'application obligatoire pour chaque État membre qui doit proposer aux agriculteurs un dispositif d'aides

\footnotetext{
${ }^{4}$ https://esrcarto.supagro.inra.fr/intranet/
} 
Author-produced version of the article published in Revue d'Economie Régionale et Urbaine (RERU), 2010, vol. 3, p. 439 - 471 The original publication is available at http://www.cairn.info/resume.php?ID_ARTICLE=RERU_103_0439

doi : $10.3917 /$ reru. 103.0439

agro-environnementales. Toutefois, comme aujourd'hui encore, les MAE sont contractualisées par les agriculteurs sur la base du volontariat. Enfin en 1999, la deuxième réforme de la PAC (Agenda 2000) conforte ce virage avec la mise en œuvre du second pilier et de son Règlement de Développement Rural (RDR) qui institutionnalise une politique européenne de développement rural.

\subsection{Un nombre très élevé de MAE déclinées régionalement}

Les mesures agro-environnementales (MAE) relèvent à la fois d'une politique communautaire dans leurs cofinancements et principes généraux mais également de politiques nationales et régionales pour tenir compte des enjeux environnementaux territorialisés et des organisations administratives propres à chaque État membre.

Au plan européen, sur la période 2000-2006, les MAE sont mises en œuvre dans le cadre du règlement de développement rural ou RDR (COMMISSION EUROPEENNE, 1999b). Il s’agit d'aides versées de manière contractuelle pour une durée de cinq ans à des agriculteurs volontaires. Elles sont destinées à compenser les coûts induits par des changements de pratiques agricoles, dans l'objectif de préserver ou améliorer les ressources naturelles (eau et sol), la biodiversité et le paysage. Il s'agit par exemple, d'un soutien à la reconversion des terres arables en prairies (pour favoriser la biodiversité) ou à la diminution des surfaces en sol nu pendant l'hiver (pour lutter contre l'érosion ou le lessivage des nitrates). L'application du RDR diffère fortement selon l'organisation institutionnelle et la répartition territoriale du pouvoir des États membres.

En France, le RDR a été transposé dans le plan de développement rural national (PDRN). Les MAE y sont regroupées en 25 actions sous forme d'un catalogue national ou « annexe B » pour atteindre les deux principaux objectifs : (1) orientation des exploitations agricoles vers une agriculture durable et (2) protection et mise en valeur du patrimoine écologique des zones du réseau Natura 2000. Ces 25 actions nationales sont elles-mêmes déclinées en 175 mesures qui précisent le cahier des charges. La France a choisi de régionaliser fortement la mise en œuvre de son PDRN puisqu'elle a permis aux 22 régions françaises d'adapter le cahier de charges national de chacune de ces mesures en fonction des contextes locaux. Au total, cet ensemble représente 2650 mesures locales pour la période 2000 - 2006 qui sont répertoriées dans 22 synthèses agro-environnementales régionales. Le tableau 
Author-produced version of the article published in Revue d'Economie Régionale et Urbaine (RERU), 2010, vol. 3, p. 439 - 471 The original publication is available at http://www.cairn.info/resume.php?ID_ARTICLE=RERU_103_0439

doi : $10.3917 /$ reru. 103.0439

2 illustre pour l'action 5 (implanter des éléments fixes du paysage) comment des mesures nationales du PDRN sont traduites dans une région.

Tableau 3: $\quad$ Exemple de l'action 5 et de ses mesures associées pour une région

\begin{tabular}{|c|c|}
\hline $\begin{array}{l}\text { Mesures nationales du PDRN } \\
\text { (Annexe B) }\end{array}$ & $\begin{array}{c}\text { Mesures régionales } \\
\text { (Synthèse agroenvironnementale régionale) }\end{array}$ \\
\hline 0501A - Plantation et entretien d'une haie & $\begin{array}{l}\text { 0501A } 01 \text { - Haie paysagère simple } \\
\text { 0501A } 02 \text { - Haie paysagère double }\end{array}$ \\
\hline $\begin{array}{l}\text { 0502A - Plantation et entretien d'un alignement } \\
\text { d'arbres ou d'arbres isolés }\end{array}$ & $\begin{array}{l}\text { 0502A01 - Alignement d'arbres } \\
\text { 0502A11 - Arbres isolés }\end{array}$ \\
\hline
\end{tabular}

Les MAE peuvent être souscrites à travers deux types de dispositifs, reposant sur des mesures contractuelles quinquennales :

- des mesures généralistes qualifiées « de masse » correspondant à des enjeux nationaux. Elles sont mises en œuvre dans le cadre d'une contractualisation simplifiée et ciblent plus particulièrement certains systèmes d'exploitation (notamment l'élevage extensif). Il s’agit de la Prime Herbagère Agro-Environnementale (PHAE), de la MAE rotationnelle et de la MAE tournesol ;

- des mesures ciblées avec le Contrat Territorial d’Exploitation (CTE), qui est une contractualisation d'un projet global d'exploitation (avec une partie économique et une partie environnementale), territorialisé par rapport aux enjeux environnementaux prioritaires. Le CTE, outil privilégié de la politique agro-environnementale française de 2000 à juin 2002 est remplacé, fin 2003, par le Contrat d’Agriculture Durable (CAD) après une année d’interruption.

\subsection{Une collecte d'indicateurs la plus exhaustive possible ...}

Pour aider à l'évaluation des mesures généralistes ou ciblées, nous avons réalisé un recensement le plus exhaustif possible des méthodes utilisant des indicateurs agroenvironnementaux. L'état de l'art international montre que ces méthodes sont très nombreuses et se sont fortement développées depuis une vingtaine d'années (RosNOBLET et al., 2006). Notre analyse a porté sur 220 méthodes, (i) spécialisées en agriculture ou appliquées au domaine rural et/ou aux milieux naturels, compte tenu de leur lien plus ou moins marqué avec les activités agricoles et (ii) publiées dans les revues référencées ou issues de rapports d’études ayant fait l'objet de validation par les mandataires publiques (comme par exemple l'indicateur 
Author-produced version of the article published in Revue d'Economie Régionale et Urbaine (RERU), 2010, vol. 3, p. 439 - 471 The original publication is available at http://www.cairn.info/resume.php?ID_ARTICLE=RERU_103_0439

doi : $10.3917 /$ reru. 103.0439

de fréquence de traitement). Ce recensement des méthodes utilisant des indicateurs a été réalisé en deux temps :

- la base de données d'indicateurs développée par l’INRA de Colmar sur les indicateurs de durabilité a été initialement mobilisée (RosNOBLET et al., 2006). Elle a été construite à partir d’une recherche bibliométrique par mots clés portant sur les termes de durabilité, méthode d’évaluation, indicateurs et leurs nombreux termes alternatifs et synonymes. Seuls les indicateurs ayant trait à l’environnement ont été retenus.

- cette base de données a été complétée par d'autres indicateurs spécifiques aux enjeux agro-environnementaux (sol, eau, biodiversité et paysages), recensés à partir de nos propres recherches bibliographiques.

L'ensemble de ces méthodes comprend 2.500 indicateurs qui ont fait l'objet d'une analyse détaillée suivant les critères présentés au paragraphe ci dessous. Ils ont tous été enregistrés dans une base de données sur les indicateurs agro-environnementaux (BIAE) spécifiquement construite pour cette recherche.

La BIAE développée rassemble à la fois les 76 indicateurs préconisés par la Commission européenne (2000) mais aussi les 2.424 autres indicateurs issus des 220 méthodes recensées. Pour chaque indicateur, 8 variables principales qualitatives discrètes sont renseignées : nom de l'indicateur, nom de la méthode, contribution de l'indicateur au dispositif, statut de l'indicateur (état, pression, suivi, impact), enjeux agro-environnementaux et lien entre enjeux et indicateur, bases de données mobilisables pour le calcul, mode de calcul et échelle spatiale. La structure organisationnelle de la BIAE (présentée en annexe 2) permet de sélectionner par tri successif les indicateurs. Il est ainsi possible de rechercher les indicateurs en fonction de leur statut (PSIR), de leurs enjeux (sol, eau, biodiversité, paysage), de leur échelle (parcelle agricole, exploitation, zonages à enjeu environnemental, zonages administratifs) et de leur calculabilité dans une des cinq bases de données expertisées (de l’ASP ou du SSP).

Une analyse de la BIAE sur le nombre d'indicateurs par méthode montre que plus de la moitié de ces 220 méthodes ne présentent qu'un seul indicateur (comme par exemple l'indicateur pesticide de fréquence de traitement (GRAVESEN, 2003)). L'autre moitié se répartit entre des méthodes comprenant de 2 à 10 indicateurs comme par exemple la méthode 
Author-produced version of the article published in Revue d'Economie Régionale et Urbaine (RERU), 2010, vol. 3, p. 439 - 471 The original publication is available at http://www.cairn.info/resume.php?ID_ARTICLE=RERU_103_0439

doi : $10.3917 /$ reru. 103.0439

INDIGO ${ }^{\circledR}$ (BOCKSTALler et al., 2006) et des méthodes en ayant plus de 10 comme IDEA (VILAIN et al., 2008).

\section{3. ... mais au final seulement 40 indicateurs mobilisables pour évaluer les effets propres des MAE biodiversité et eau}

Pour les enjeux eau (qualité et quantité) et biodiversité, les 220 méthodes recensent 1.289 indicateurs (55\% pour la biodiversité et 45\% pour l'eau), soit un peu plus de moitié des indicateurs de la BIAE.

En appliquant le critère 1 - lien fort avec l'enjeu - seuls les indicateurs ayant un degré de lien fort avec l'enjeu retenu (cf. Tableau en Annexe 1) sont conservés. Un peu plus d’un tiers des indicateurs ne répondent pas à ce critère, ce qui ramène leur nombre à 836.

Le critère 2 sur le statut de l'indicateur dans la chaîne causale des effets est le plus radical, puisqu'il ne permet de retenir que 165 indicateurs de pression. Dans beaucoup de méthodes recensées, ce sont les indicateurs de suivi (R, response) qui sont mobilisés (cf. Tableau 1) et très largement utilisés dans les évaluations des politiques, ce qui explique cette inflexion nette du nombre d'indicateurs, quand on applique ce $2^{\text {ème }}$ critère.

L'application du critère 3 montre qu'une majorité d'indicateurs est calculable aux échelles administratives (communes, département, région ou nation), alors que seulement 84 le sont à l'échelle de l'exploitation, qui est celle retenue dans la méthode de mesure des effets propres.

Enfin, l'application du critère $\mathbf{4}$ conduit à réduire de moitié les indicateurs mobilisables. La base de l'ASP ne dispose que des données de suivi du programme, elle ne comprend aucun indicateur de pression. Seules les bases de données du SSP ont des variables pertinentes. Il s'agit principalement du Recensement de l'Agriculture (RA), qui permet de calculer une bonne partie des indicateurs sur la biodiversité, ainsi que de l'enquête Pratiques agricoles (PK), qui, elle, est plus mobilisée dans le calcul des indicateurs sur l'eau. Toutes ces données sont disponibles à l'échelle de l'exploitation agricole et ont également un sens lorsqu’elles sont agrégées aux échelles administratives.

Au final, l'application de ces quatre critères fait ressortir 40 indicateurs susceptibles d'être utilisés dans les méthodes d'évaluation des effets propres des MAE (cf. figure 2). 
Author-produced version of the article published in Revue d'Economie Régionale et Urbaine (RERU), 2010, vol. 3, p. 439 - 471 The original publication is available at http://www.cairn.info/resume.php?ID_ARTICLE=RERU_103_0439 doi : $10.3917 /$ reru.103.0439

Figure 2: $\quad$ Application des 4 critères de sélection sur les indicateurs eau et biodiversité

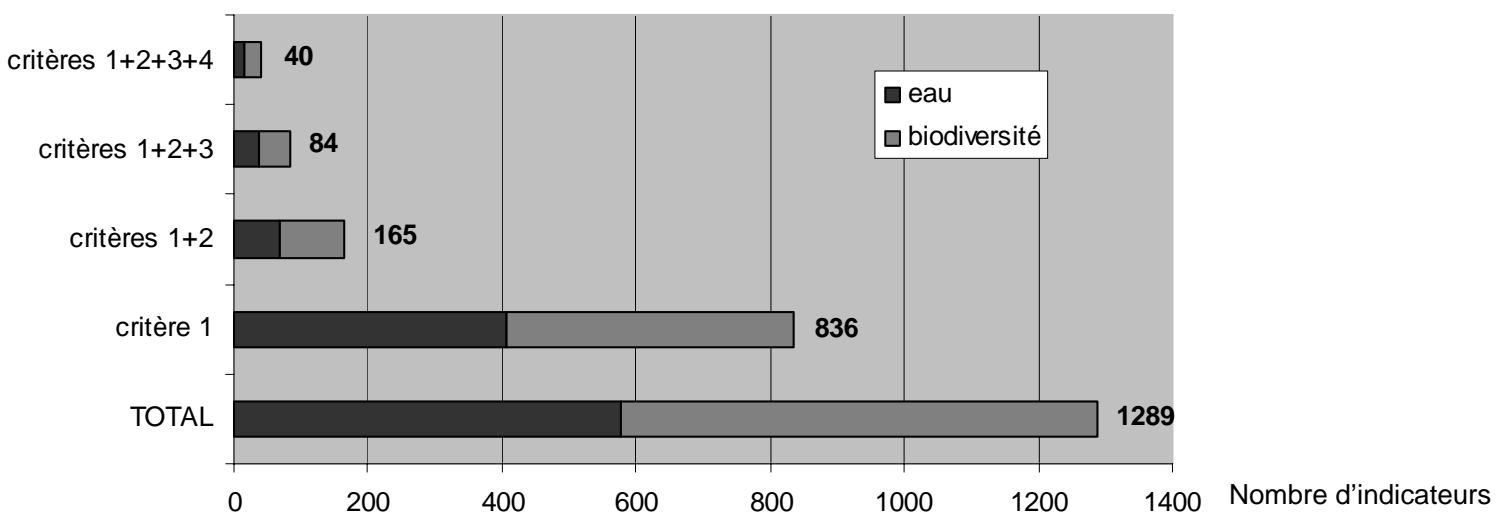

\begin{tabular}{|l|l|}
\hline Critère 1 & Intensité de la relation avec l'enjeu et la pratique (faible, moyen, fort) \\
\hline Critère 2 & Statut de l'indicateur (état, suivi, pression, impact) \\
\hline Critère 3 & $\begin{array}{l}\text { échelle de calcul (parcelle, exploitation, zonages environnementaux, } \\
\text { zonages administratifs) }\end{array}$ \\
\hline Critère 4 & Variable disponibles dans les bases de données publiques expertisées \\
\hline
\end{tabular}

Ces 40 indicateurs peuvent être analysés (1) en les confrontant aux MAE réellement souscrites sur la période 2000-2006 (analyse de la cohérence d'ensemble) et (2) en interrogeant leur capacité à rendre compte de l’impact environnemental des pratiques.

Sur le premier point, les 40 indicateurs se répartissent en 21 indicateurs à enjeu biodiversité et 19 à enjeu eau (cf. annexe 3). Le tableau 3 met en perspective les principales mesures contractualisées (pour les surfaces totales engagées supérieures à 150.000 ha) avec les enjeux principaux correspondants et les indicateurs susceptibles d'être retenus pour l'évaluation.

Tableau 4: MAE les plus contractualisées, enjeux et indicateurs correspondants

\begin{tabular}{|c|l|c|c|c|}
\hline \multicolumn{2}{|c|}{$\begin{array}{c}\text { Code et intitulé de la MAE } \\
\text { sur la période 2000 - 2006 }\end{array}$} & $\begin{array}{c}\text { Surfaces } \\
\text { engagées } \\
\text { (en ha) }\end{array}$ & $\begin{array}{c}\text { Enjeux } \\
\text { principaux }\end{array}$ & $\begin{array}{c}N^{\circ} \text { de } \\
\text { l'indicateur } \\
\text { (annexe 3) }\end{array}$ \\
\hline 20PPP & Gestion extensive des prairies permanentes / PHAE & 1695690 & BIOr2, BIOc4 & 16,18 \\
\hline 2001A & Gestion extensive de la prairie par la fauche / PHAE & 833290 & BIOr2, BIOc4 & $16,18,15$ \\
\hline 0903A & Adapter la fertilisation en fonction des analyses de sols & 692700 & EAUn1 & 22 \\
\hline
\end{tabular}


Author-produced version of the article published in Revue d'Economie Régionale et Urbaine (RERU), 2010, vol. 3, p. 439 - 471 The original publication is available at http://www.cairn.info/resume.php?ID_ARTICLE=RERU_103_0439 doi : $10.3917 /$ reru.103.0439

\begin{tabular}{|c|c|c|c|c|}
\hline & $\begin{array}{l}\text { Code et intitulé de la MAE } \\
\text { sur la période } 2000 \text { - } 2006\end{array}$ & $\begin{array}{l}\text { Surfaces } \\
\text { engagées } \\
\text { (en ha)* }\end{array}$ & $\begin{array}{c}\text { Enjeux } \\
\text { principaux }\end{array}$ & $\begin{array}{c}\mathrm{N}^{\circ} \text { de } \\
\text { l'indicateur } \\
\text { (annexe 3) }\end{array}$ \\
\hline 20PPT & Gestion extensive des prairies temporaires & 624720 & BIOc4 & $11,16,18$ \\
\hline 0205A & Mesure rotationnelle & 624580 & BIOc2 & $2,3,4,5,6$ \\
\hline 0801A & Lutte raisonnée & 577300 & EAUp1 & 31, 32, 33 \\
\hline $\begin{array}{l}19 \\
\text { PHA }\end{array}$ & $\begin{array}{l}\text { Maintien de l'ouverture des espaces à gestion extensive / } \\
\text { PHAE }\end{array}$ & 512180 & BIOr2, BIOc4 & 15 \\
\hline 0301A & $\begin{array}{l}\text { Implantation de cultures intermédiaires en période de } \\
\text { risque }\end{array}$ & 232900 & EAUn2 & 28,29 \\
\hline $2001 C$ & $\begin{array}{l}\text { Gestion extensive de la prairie par la fauche avec } \\
\text { suppression fertilisation organique }\end{array}$ & 201507 & $\begin{array}{l}\text { EAUn1, BIOc4, } \\
\text { BIOr2 }\end{array}$ & $\begin{array}{l}11,15,16,18, \\
23\end{array}$ \\
\hline 1903A & $\begin{array}{l}\text { Maintien de l'ouverture des espaces à gestion extensive } \\
\text { (estives, alpages, parcours...) }\end{array}$ & 161387 & BIOr2 & $11,16,18$ \\
\hline 1303A & Travail du sol simplifié (zéro labour) & 154854 & EAUn2, EAUp2 & aucun \\
\hline
\end{tabular}

* surfaces contractualisées 2000 - 2006 (AND, 2009)

Il ressort de cette analyse qu'il est possible de pouvoir disposer d'indicateurs calculables pour la plupart des MAE que ce soient pour les enjeux biodiversité et eaux, à l'exception de la MAE «travail du sol simplifié » pour laquelle aucun indicateur pertinent n'est calculable.

La capacité de ces 40 indicateurs à rendre compte de l'impact environnemental des pratiques (second point) est analysée sous l’angle de la causalité en étudiant le statut de chaque indicateur dans la chaîne causale des effets (voir section 2.2). Le degré de causalité est apprécié à partir d'une grille d'analyse qualitative comprenant trois modalités (faible, moyen, fort) qui rendent compte de la présence d'une valeur de référence pour chaque indicateur. En effet, calculer la valeur absolue d'un indicateur n’a pas de signification en soi. Pour interpréter les résultats, il importe de pouvoir la comparer à une valeur de référence (VAN CAUWENBERGH et al., 2007). Le degré de causalité est considéré comme :

- faible, lorsque l'indicateur est construit sur les pratiques (voir section 2.2) avec une absence de valeur de référence,

- moyen, lorsque l'indicateur est construit, soit sur les pratiques et dispose d'une de valeur de référence, soit sur les émissions mais ne présente pas de valeur de référence, 
Author-produced version of the article published in Revue d'Economie Régionale et Urbaine (RERU), 2010, vol. 3, p. 439 - 471 The original publication is available at http://www.cairn.info/resume.php?ID_ARTICLE=RERU_103_0439 doi : $10.3917 /$ reru. 103.0439

- élevé, lorsque l'indicateur est construit sur les émissions et doté d’une valeur de référence.

Au final, chaque indicateur dispose d'un niveau de causalité (cf. annexe 3), qui peut être interprété également par enjeux (cf. tableau 4). Les résultats montrent que (i) les trois indicateurs présentant un degré de causalité élevé concernent l’enjeu eau et que (ii) la majorité des indicateurs présente un degré de causalité moyen.

Tableau 5: Nombre d’indicateurs calculables par enjeux, pratiques et degré de causalité

\begin{tabular}{|c|c|c|c|c|c|c|}
\hline & \multirow{2}{*}{ Code } & \multirow{2}{*}{ Pratiques agricoles } & \multirow{2}{*}{$\begin{array}{c}\text { Nombre } \\
\text { d'indicateurs }\end{array}$} & \multicolumn{3}{|c|}{ Degré de causalité } \\
\hline & & & & faible & moyen & élevé \\
\hline \multirow{7}{*}{ 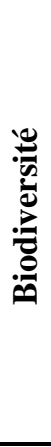 } & BIOc1 & réduire les intrants & 1 & 1 & & \\
\hline & BIOc2 & rotation des cultures & 7 & 1 & 6 & \\
\hline & BIOc3 & végétation en période critique & 0 & & & \\
\hline & BIOc4 & gestion écologique & 9 & 3 & 6 & \\
\hline & BIOr1 & $\begin{array}{l}\text { conservation des espèces faunistiques et floristiques } \\
\text { remarquables }\end{array}$ & 0 & & & \\
\hline & BIOr2 & conservation des habitats & 1 & & 1 & \\
\hline & BIOr3 & $\begin{array}{l}\text { conservation des races domestiques animales et } \\
\text { végétales menacées }\end{array}$ & 3 & 2 & 1 & \\
\hline \multirow{6}{*}{ 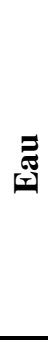 } & EAUn1 & réduire les apports (nitrate) & 2 & & 2 & \\
\hline & EAUn2 & réduire les transferts (nitrate) & 6 & 1 & 4 & 1 \\
\hline & EAUp1 & réduire les apports (pesticides) & 4 & 1 & 3 & \\
\hline & EAUp2 & réduire les transferts (pesticides) & 1 & 1 & & \\
\hline & EAUq1 & réduire les surfaces en cultures irriguées & 3 & 2 & 1 & \\
\hline & EAUq2 & réduire le niveau d'irrigation à l'hectare & 3 & & 1 & 2 \\
\hline & & Total & 40 & 12 & 25 & 3 \\
\hline
\end{tabular}

\subsection{Discussion}

Nous proposons de revenir sur nos travaux en discutant d'abord notre démarche de sélection des indicateurs puis nos résultats et perspectives de travaux complémentaires.

La démarche générale de sélection des indicateurs pose la question des conditions dans lesquelles les critères retenus ont été proposés mais aussi de leur application à chacun des indicateurs expertisés. Pour formaliser cette étape essentielle du choix des critères, nous avons réuni à deux reprises un groupe d'experts de la recherche chargés de discuter les premières propositions établies par les auteurs. Par analogie au processus complet de validation d'indicateurs (BOCKSTALLER et GIRARDIN, 2003), cette validation a permis de vérifier (i) le 
Author-produced version of the article published in Revue d'Economie Régionale et Urbaine (RERU), 2010, vol. 3, p. 439 - 471 The original publication is available at http://www.cairn.info/resume.php?ID_ARTICLE=RERU_103_0439

doi : $10.3917 /$ reru. 103.0439

bien fondé de la démarche scientifique («well founded») et (ii) la réalisation des objectifs («achieving the overall objectives »). Quant à l'application des quatre critères sur chaque indicateur présent dans la base de données, la question la plus délicate a porté sur les critères 1 et 2. L'intensité du lien (critère 1, note de 1 à 3) et le statut de l'indicateur (critère 2) ont été établis systématiquement à partir des publications scientifiques se rapportant à l'indicateur. C’est lorsque les informations dans la publication n’étaient pas suffisantes que cette qualification du lien ou du statut a été déterminée par consultation d'experts associés à notre travail ou bien à partir de notre propre expertise liée à nos travaux précédemment conduits.

Quant au très faible nombre d’indicateurs (40), il s'explique en partie pour les raisons suivantes :

- les indicateurs analysés ont été majoritairement développés dans les années 1980 à 2000. Durant cette période, les lignes directrices de l'évaluation des politiques publiques agro-environnementales n’étant pas établies, les indicateurs ne pouvaient pas avoir comme objectifs principaux de répondre aux quatre critères de sélection proposés,

- de nombreux indicateurs pertinents ont été développés dans l’objectif d'une évaluation d’une «démarche de progrès » de l'exploitant pour rendre compte et suivre la performance environnementale individuelle des pratiques agricoles. Or cette évaluation individuelle nécessite une enquête auprès de chaque agriculteur pour recueillir des données contextuelles telles que la localisation des parcelles, la nature pédologique des sols, les caractéristiques toxicologiques des pesticides, les infrastructures écologiques présentes. De telles variables ne sont pas encore disponibles dans les bases de données nationales.

- les méthodologies d'évaluation économique des effets propres appliquées à l’agroenvironnement ne sont pas encore stabilisées compte tenu de leur relative jeunesse. Aussi, ces méthodes sont encore peu, voir très peu connues de la communauté scientifique qui travaille sur les indicateurs. Il parait logique que les indicateurs susceptibles de pouvoir être couplés à ces méthodes économétriques n’aient pas été une des priorités des travaux sur les indicateurs agro-environnementaux.

Ces travaux ont également permis la création d'une base de données très complète d'indicateurs (BIAE), permettant de pouvoir établir des listes d'indicateurs par enjeu 
Author-produced version of the article published in Revue d'Economie Régionale et Urbaine (RERU), 2010, vol. 3, p. 439 - 471 The original publication is available at http://www.cairn.info/resume.php?ID_ARTICLE=RERU_103_0439

doi : $10.3917 /$ reru. 103.0439

environnemental (eau, sol, biodiversité, etc.). Cette dernière pourrait évoluer vers une métabase ouverte en ligne permettant de trier les indicateurs en fonction des quatre critères présentés ou d’autres qu’il conviendrait alors de compléter.

Par ailleurs, malgré le grand nombre de méthodes étudiées, ces résultats sont susceptibles d'évoluer dans les prochaines années car :

- les travaux scientifiques sur les indicateurs s’enrichissent chaque année et de nouveaux indicateurs pourraient être considérés comme pertinents dans quelques années en appliquant le filtre de nos quatre critères,

- les variables des différentes bases de données statistiques ou administratives, elles non plus, ne sont pas figées dans le temps. Le contenu des bases de données étudiées évolue également en fonction des besoins nouveaux exprimés par l'administration ou les autres utilisateurs.

- les dispositifs réglementaires évalués sont eux aussi évolutifs. Ainsi sur la prochaine période de programmation du développement rural 2007-2013 (PDRH, 2008), la France a retenu un nombre de MAE beaucoup plus restreint avec des objectifs mesurés par des indicateurs de pression (notamment l'indicateur de fréquence de traitement pour les MAE à enjeux réduction de pesticides). Ces nouveautés sont susceptibles de modifier nos résultats. Par ailleurs, le récent bilan de santé de la PAC (COMMISSION EUROPEENNE, 2009) a élargi les MAE aux questions environnementales du changement climatique. Certains des indicateurs d'évaluation de la performance énergétique recensés dans la BIAE (BOCHU, 2002 ; VILAIN et al., 2008) pourraient devenir calculables si le MAAP décidait d’élargir, dans les bases de données publiques, la collecte des informations aux consommations énergétiques des exploitations.

Enfin, notre travail nous amène à nous questionner sur deux points concernant la transposition de cette démarche à l'évaluation des MAE de la programmation 2007-2013. Le premier point pose la question des indicateurs idéaux qu'il conviendrait de mettre à disposition des évaluateurs. Concernant l'eau et la biodiversité, les objectifs de cette nouvelle programmation sont dans la continuité : restauration ou préservation de la ressource l'eau en appui aux objectifs de la Directive Cadre sur l'Eau et préservation de la biodiversité dans les zones Natura 2000. 
Author-produced version of the article published in Revue d'Economie Régionale et Urbaine (RERU), 2010, vol. 3, p. 439 - 471 The original publication is available at http://www.cairn.info/resume.php?ID_ARTICLE=RERU_103_0439 doi : $10.3917 /$ reru. 103.0439

S’agissant de la biodiversité, un indicateur pertinent serait l'indicateur "présence d’infrastructures agro-écologiques » qui a été retenu pour le niveau 3 de la certification à haute valeur environnementale des exploitations agricoles suite aux travaux du comité opérationnel (COMOP) du Grenelle environnement ${ }^{5}$ (COMOP, 2009). Cet indicateur présente de très nombreux avantages : il est validé socialement par les acteurs ayant participé au COMOP, il est issu de travaux scientifiques sur le rôle des infrastructures agro-écologiques dans la préservation de la biodiversité et enfin il dispose d’une valeur seuil (au minimum 10 \% de la SAU en infrastructures écologiques). Les infrastructures agro-écologiques prises en compte sont celles retenues dans le cadre des « bonnes conditions agricoles et environnementales » de la conditionnalité des aides de la PAC. Un système de pondération définit, pour chaque type d'infrastructures ${ }^{6}$, une surface de biodiversité équivalente pour donner une valeur finale de biodiversité exprimée dans une unité commune. Ces variables font aujourd’hui défaut dans les bases de données analysées (à l’exception des bandes enherbées).

Quant à l'évaluation des pratiques phytosanitaires sur la qualité de l'eau, l'indicateur phytosanitaire idéal devrait intégrer dans sa construction des variables sur les pratiques agricoles (dates, localisation et nombre de traitements), mais il devrait également tenir compte de variables concernant le degré d'exposition (résultant de la dispersion et de la concentration du pesticide dans l'environnement) (VAN DER WERF et ZIMMER, 1998), des caractéristiques des matières actives (solubilité, volatilité, toxicité) et des caractéristiques des sols (FALCONER, 2002). La majorité de ces variables ne sont et ne seront pas à court terme encore disponibles dans les bases de données analysées. A défaut, nous considérons que l’indicateur de fréquence de traitement (PINGAUlt et al., 2009) serait susceptible de pouvoir être retenu à la condition d’intégrer les travaux en cours sur les seuils de référence (COMOP, 2009). Toutefois, pour être mobilisée dans une évaluation nationale des MAE, la base de sondage de l'enquête " pratiques culturales » devra être élargie, pour (i) la rendre représentative non plus des pratiques de la culture sur la parcelle mais des pratiques de la culture de l'exploitation enquêtée, et (ii) intégrer suffisamment de bénéficiaires de MAE.

\footnotetext{
${ }^{5}$ Voir http://agriculture.gouv.fr/sections/magazine/focus/certification

${ }^{6}$ haies, bandes enherbées, vergers haute tige, jachère mellifère, tourbières, arbres isolés, fossés, mares, lavognes, murets, prairies permanentes, landes, parcours
} 
Author-produced version of the article published in Revue d'Economie Régionale et Urbaine (RERU), 2010, vol. 3, p. 439 - 471 The original publication is available at http://www.cairn.info/resume.php?ID_ARTICLE=RERU_103_0439 doi : $10.3917 /$ reru. 103.0439

Le second point interroge la question des contraintes fortes mise en avant dans ces travaux pour l'accès aux données nécessaires à la construction du contrefactuel. Comment lever une partie de cet obstacle pour avancer ? Pour réaliser ce type de traitements économétriques, la base de données des exploitants agricoles devra comporter suffisamment de bénéficiaires et de non bénéficiaires, avant et après la mise en place du programme. Pour chaque agriculteur, il est nécessaire de connaître simultanément des données sur le niveau des pratiques avant et après le lancement de la MAE, ainsi que ses caractéristiques observables avant le lancement de la politique (de sorte que le niveau de ces variables ne soit pas influencé par la politique elle-même). Ces conditions imposent d'apparier au moins deux enquêtes nationales (Pratiques culturales et Recensement Agricole). Aujourd’hui, compte tenu du très faible nombre de MAE souscrites et de la forte territorialisation d'une partie de ces mesures (les MAE territorialisées), nous considérons qu'il est peu probable que les deux approches contrefactuelles mises en avant (matching ou double différence) puissent être appliquées de façon opérationnelle lors de la prochaine évaluation prévue en 2013. Par contre, il serait pertinent et scientifiquement intéressant d'utiliser ce type d'approche dans de nouveaux travaux de recherche pour conduire l'évaluation d'une mesure à caractère national : la conversion à l'agriculture biologique. Ces travaux impliquent notamment de (i) travailler avec le MAAP au plan méthodologique sur une modification de la représentativité de l'échantillon de l'enquête Pratiques Culturales pour qu'elle contienne le maximum de bénéficiaires des mesures de conversion à l'agriculture biologique dès la prochaine enquête et (ii) proposer les indicateurs pertinents par rapport aux spécificités de l’agriculture biologique.

\section{CONCLUSION}

Le cadre communautaire de l'évaluation des MAE sur la période 2000-2006 impose aux États membres de répondre à des questions d'évaluation communes. Notre analyse montre que les indicateurs proposés par la Commission européenne sont essentiellement des indicateurs pour rendre compte du suivi (indicateurs de reporting) des programmes nationaux afin d’être au final agrégés à l'échelon européen. Ils ne peuvent pas rendre compte de l'impact environnemental des MAE dans le cadre d’une évaluation qui s’intéresse aux effets propres du soutien à l'agro-environnement. Nous montrons que les indicateurs pertinents pour mesurer ces effets propres doivent répondre aux quatre critères suivants : (i) être capable d'évaluer au moins un des trois enjeux agro-environnementaux et ses pratiques agricoles associées, (ii) être un indicateur de pression, (iii) avoir un sens à l'échelle de l'exploitation agricole et (iv) être 
Author-produced version of the article published in Revue d'Economie Régionale et Urbaine (RERU), 2010, vol. 3, p. 439 - 471 The original publication is available at http://www.cairn.info/resume.php?ID_ARTICLE=RERU_103_0439 doi : $10.3917 /$ reru. 103.0439

calculable dans les bases de données nationales pour lesquelles un appariement entre elles est possible.

Une base de données de 2.500 indicateurs a été développée, à partir de l’analyse de 220 méthodes. Le traitement de cette base de données à partir de ces quatre critères montre que sur les 1290 indicateurs caractérisant les deux enjeux de préservation de la biodiversité ou de la ressource en eau, seulement 21 indicateurs pour la biodiversité et 19 pour l'eau sont aujourd’hui mobilisables pour évaluer les effets propres des MAE eau et biodiversité.

Ce très faible nombre d'indicateurs susceptibles d'être intégrés dans des méthodes économiques d'évaluation des effets propres s'explique en partie pour les raisons suivantes : (1) l'obligation réglementaire de l'évaluation des dispositifs agro-environnementaux est récente (une dizaine d’année), (2) les dispositifs réglementaires nationaux connaissent de nombreuses évolutions peu propices à la stabilisation de méthodes quantitatives d'évaluation, (3) les méthodologies d'estimation des effets appliquées à l'agro-environnement sont en cours de stabilisation, (4) l’appropriation par les décideurs publics des travaux scientifiques sur les indicateurs n’a commencé à se généraliser qu’à partir des années 2000.

Sur la nouvelle période de programmation 2007-2013 (COMMISSION EUROPEENNE, 2005), le nouveau Cadre Commun de Suivi et d'Évaluation (CCSE) constitue le référentiel pour le suivi et l'évaluation des MAE. Il serait utile de saisir l'opportunité prévue par ces nouvelles dispositions qui permettent « de définir des indicateurs supplémentaires dans le cadre des programmes. Ces indicateurs supplémentaires devraient être développés par les États membres et les partenaires de programme d'une manière souple, mais conforme aux principes généraux qui régissent l'utilisation d'indicateurs dans le CCSE »(COMMISSION EUROPEENNE, 2006, page 2). C'est pourquoi, pour accompagner ces perspectives de travaux de recherche présentées en discussion, nous proposons qu'une réflexion nationale s’engage sur la place des variables agro-environnementales dans les données de la statistique publique. Ce point ouvre plus largement la discussion sur les modalités d’une adaptation des enquêtes de la statistique publique aux nouveaux enjeux de la PAC. 
Author-produced version of the article published in Revue d'Economie Régionale et Urbaine (RERU), 2010, vol. 3, p. 439 - 471 The original publication is available at http://www.cairn.info/resume.php?ID_ARTICLE=RERU_103_0439 doi : 10.3917/reru.103.0439

\section{REMERCIEMENTS}

Les auteurs remercient Philippe GIRARDIN pour la mise à disposition de la base de données du projet ADD (INRA Colmar) et Jérôme ROSNOBLET pour son appui à la mise à jour de la base de données.

\section{Bibliographie}

AND, 2009, Évaluation ex-post du PDRN. Partie sur le « soutien à l'agro-environnement ». Rapport final, Ministère de l’Agriculture et de la Pêche, Paris.

Behaghel L., Crepon B., GuRgand M., 2009, Évaluation d'impact de l'accompagnement des demandeurs d'emploi par les opérateurs privés de placement et le programme Cap vers l'entreprise, Rapport final, 68 p.

Benabou R, Kramarz, F., Prost C., 2004, «Zones d'éducation prioritaires : quels moyens pour quels résultats ? Une évaluation sur la période 1982-1992 », Economie et Statistique, vol. 380, pp. 3-34.

Bénabou R., Kramarz F., Prost C., 2009, « The French zones d'éducation prioritaires : Much ado about nothing ? », Economics of Education Review, vol. 28, pp. 345-356.

Bochu J.-L., 2002, PLANETE : méthode pour l'analyse énergétique de l'exploitation agricole et l'évaluation des émissions de gaz à effet de serre, Solagro, Toulouse.

Bockstaller C. et Girardin P., 2003, « How to validate environmental indicators ? », Agricultural Systems, vol. 76, pp. 639-653.

Bockstaller C. et GiRARDin P., 2006, « Evaluation agri-environnementale des systèmes de culture : la méthode INDIGO® », Oléoscope, n85, pp.4-6.

Bockstaller C., Girardin P., VAn Der Werf H, 1997, « Use of agro-ecological indicators for the evaluation of farming systems », European Journal of Agronomy, vol. 7, pp. 261-270.

Bockstaller C., Guichard L., David M., Aveline A., Girardin P., PlantureuX S., 2008, «Agri-environmental indicators to assess cropping and farming systems. A review », Agronomy for Sustainable Development, vol. 28, pp. 139-149.

BonNieux F., 2009, Bilan critique de la politique agri-environnementale et perspectives d'évolution, in Aubert F., Piveteau V., Schmitt B. (eds), Politiques agricoles et territoires, Ed QUAE, Versailles, pp. 141-163. 
Author-produced version of the article published in Revue d'Economie Régionale et Urbaine (RERU), 2010, vol. 3, p. 439 - 471 The original publication is available at http://www.cairn.info/resume.php?ID_ARTICLE=RERU_103_0439

doi : $10.3917 /$ reru. 103.0439

BouRguignon F., 2009, « Rapport final sur l'évaluation des expérimentations RSA », Comité d’Évaluation des expérimentations, Haut commissaire pour la solidarité active contre la pauvreté, La documentation française, Paris.

Brodaty T., Crepon B., Fougeres D., 2002, « Les méthodes micro-économétriques d'évaluation : développements récents et applications aux politiques actives de l'emploi », 15ème Congrès des Économistes Belges de Langue Française, Namur, 24 p.

Chabe-Ferret S., Subervie J., 2008, Assistance méthodologique à la préparation de l'évaluation ex-post du PDRN 2000-2006 en matière de soutien à l'agroenvironnement Proposition d'une méthode d'estimation des effets propres des mesures agroenvironnementales et de l'ICHN, Rapport Cemagref, 66 p.

Clergue B., Amiaud B., Pervanchon F., Lasserre-Joulin F., Plantureux S., 2005, « Biodiversity : function and assessment in agricultural areas. A review », Agronomy Sustainable Development, n²5, pp. 1-15.

COMMission EuRopeEnNE, 1999 a, Evaluer les programmes socio-économiques. Conception et conduite d'une évaluation : choix et utilisation des indicateur pour le suivi et l'évaluation. Office des publications officielles des communautés européennes - collection MEANS, volumes 1, 2 et 3, Bruxelles.

Commission EuropeEnNe, 1999b, Règlement $\mathrm{N}^{\circ} 1257 / 99$ concernant le soutien au développement rural par le Fonds européen d'orientation et de garantie agricole, JOCE L 160 du 26 juin 1999, 41 p.

COMMISSION EuROPEENNE, 2000, Questions d'évaluation commune accompagnées de critères et d'indicateurs. Evaluation des programmes de développement rural pour la période 20002006 soutenus par le FEOGA. Doc VI/12004/Final, 56 p.

Commission Europeenne, 2005, Règlement du Conseil (CE) nº1698/2005 concernant le soutien au développement rural par le Fonds européen agricole pour le développement rural (Feader), JOCE L 277, 40 p.

COMMISSION EuRopeEnNE, 2006, Note d'orientation A : choix et utilisation d'indicateurs, issu du manuel relatif au cadre commun de suivi et d'évaluation, 8 p.

Commission EuROPEEnNE, 2009, Règlement (CE) N74/2009 DU CONSEIL du 19 janvier 2009 portant modification du règlement (CE) no 1698/2005 concernant le soutien au 
Author-produced version of the article published in Revue d'Economie Régionale et Urbaine (RERU), 2010, vol. 3, p. 439 - 471 The original publication is available at http://www.cairn.info/resume.php?ID_ARTICLE=RERU_103_0439 doi : $10.3917 /$ reru. 103.0439

développement rural par le Fonds européen agricole pour le développement rural, JOCE L 30 du 3001 2009, 12 p.

COMOP, 2009, La certification environnementale des exploitations, Rapport du COMOP du 15 juin 2009 et annexes, Ministère de l’Alimentation, de l’Agriculture et de la Pêche, 16 p.

Convention on Biological Diversity, 2000, Decision V/5 - Agricultural biological diversity: review of phase I of the programme of work and adoption of a multi-year work programme. Annex III, Decisions adopted by the Conference of Parties to the Convention on Biological Diversity (fifth meeting).

CORPEN, 1988, Bilan de l'azote à l'exploitation, CORPEN, Paris.

Corpen, 2003, Des indicateurs pour des actions locales de maîtrise des pollutions de l'eau d’origine agricole : éléments méthodologiques- Application aux produits phytosanitaires, CORPEN, Paris.

CORPEn, 2006, Des indicateurs azote pour gérer des actions de maîtrise des pollutions à l'échelle de la parcelle, de l'exploitation et du territoire, Groupe azote indicateurs, CORPEN, Paris.

Crepon B., Desplatz R., 2001, «Une nouvelle évaluation des effets des allégements de charges sociales sur les bas salaires », Economie et Statistique, vol. 348, n8, pp. 3-34.

Devillers J., Farret R., Girardin P., Keichinger O., Riviere J-L, Soulas G., 2005, Indicateur pour évaluer les risques liés à l'utilisation des pesticides, Lavoisier, Editions Tec et Doc, Paris.

Donnelly A., Jones M., O'MAHONY T., BYRne G., 2007, « Selecting environmental indicator for use in strategic environmental assessment », Environmental Impact Assessment Review, vol. $27, \mathrm{n}^{\circ} 2$, pp. 161-175.

EEA, 1999, Technical report No 25, Environmental indicator : Typology and overview, European Environment Agency by Edith Smeets and Rob Weterings, 19 p.

EUROPEAN ENVIRONMENT AgENCY, 2005, Agriculture and environment in EU-15 : the IRENA indicator report, Report No 6/2005

EVEN K. et KLEIN T., 2007, «Les contrats et stages aidés : un profit à moyen terme pour les participants ? Les exemples du CIE, CES et du Sife », Économie et statistique, n408-409, pp. 33-43.

FADEAR, 1999, Charte de l'Agriculture Paysanne - Grille d'analyse, 26 p. 
Author-produced version of the article published in Revue d'Economie Régionale et Urbaine (RERU), 2010, vol. 3, p. 439 - 471 The original publication is available at http://www.cairn.info/resume.php?ID_ARTICLE=RERU_103_0439

doi : $10.3917 /$ reru. 103.0439

FALCONER K., 2002, «Pesticide environnemental indicators and environnemental policy », Journal of environnemental management, vol. 62, pp. 285-300.

FJELlSTAD W., 2004, Linking farm management to effects on biodiversity and landscape, OCDE expert meetings on farm managements indicators and the environment, Expert Meeting on Farm Management Indicators and the Environment, New Zealand, 11 p.

GIBERT P., 2003, «L'évaluation de politique : contrôle externe de la gestion publique ? » Revue Française de Gestion, vol. 6, n¹47, pp. 259-273.

GIRARDiN P., BocKSTALleR C., 1997, Les indicateurs agro-écologiques, outils pour évaluer des systèmes de culture Oléagineux, Corps Gras, Lipides, Dossier : développement durable et recherches agronomiques, vol. 4 , $n^{\circ} 6$, pp. 418-426

Girardin P., Bockstaller C., VAN Der WerF H.M.G., 1999, «Indicators : Tools to Evaluate the Environmental Impacts of Farming systems », Journal of Sustainable Agriculture, vol. 13, n4, pp. 5-21.

Gras R., Benoit M., Deffontaines J.P., Duru M., LANGlet A., 1989, Le fait technique en agronomie, activités agricoles, concepts et méthodes d'étude, INRA-éditions, L'Harmattan, Paris.

Gravesen L., 2003, The Treatment Frequency Index : an indicator for pesticide use and dependency as well as overall load on the environment, Pesticide Action Network Europe, Pure conference, Copenhague, pp. 28-30.

Grosjean E., 2000, Le Portrait Agri-environnemental de l’Exploitation Agricole. Rapport au Comité d’accompagnement, Belgique, 26 p.

GUERIN M et RUAS J.F. (avec la collaboration de LETURQ, F.), 2006, «Analyse des pratiques d'évaluation des politiques agricoles et de développement rural », Notes et études économiques, n²6, pp. 35-126.

GuERIN M, 2008, «Évaluation des politiques de développement rural. Quelques éléments d’analyse », Economie Rurale, n 307, pp. 39-52.

Heckman J.J, Lalonde R., Smith J., 1999, The Economics and Econometrics of Active Labor Market Programs, in : Ashenfelter O., CARD D. (eds), Handbook of Labor Economics, vol. 3, North Holland, pp. 1865-2097.

INSTITUT INTERNATIONAL DU DÉVELOPPEMENT DURABLE, 2002, The Dashboard of Sustainability, Consultative Group on Sustainable Development Indicators, Canadian IISD 
Author-produced version of the article published in Revue d'Economie Régionale et Urbaine (RERU), 2010, vol. 3, p. 439 - 471 The original publication is available at http://www.cairn.info/resume.php?ID_ARTICLE=RERU_103_0439

doi : $10.3917 /$ reru. 103.0439

LeVrel H., 2006, Biodiversité et développement durable : quels indicateurs ? Thèse pour l’obtention du titre de docteur de l’EHESS, Spécialité : Economie Ecologique, Muséum National d'Histoire Naturelle, 406 p.

LOFREDI P., 2007, Evaluation économétrique des effets de traitement et programmes de développement à ciblages géographique. Le cas des interventions économiques en faveur du développement rural, Thèse soutenue à l'université de Bourgogne sous la direction de $\mathrm{B}$. SCHMITT.

LOFRedi P., Schmitt B, 2005, Appui méthodologique à l'évaluation du développement des zones rurales. Note sur l'évaluation des effets propres, Fascicule 3, Centre CESAER, Dijon.

LÜTZ M. et FELICI F., 2009, « Indicators to identify the agricultural pressures on environmental functions and their use in the development of agri-environmental measures ", Regional Environmental Change, vol. 9, pp. 181-196.

MagnaC, T., 2000, «L'apport de la microéconométrie à l'évaluation des politiques publiques », Cahier d'économie et de sociologie rurales, vol. 54, pp. 90-113.

Maljean J.F., Brouckaert V., Van Cauwenbergh N., Peeters A., 2004, Assessment, monitoring, implementation and improvement of farm management for environmental and sustainable agriculture purposes : a Belgian Perspective, SAFE, Expert Meeting on Farm Management Indicators and the Environment, New Zealand, 32 p.

MAurizi B. et VerRel J.-L., 2002, “Des indicateurs pour des actions de maîtrise des pollutions d'origine agricole”, Ingénieries - EAT, vol. 30, pp. 3-14.

Mayrhofer P., Steiner C., GÄrber E., Gruber E., 1996, Regionalprogramm, Ökopunkte Niederösterreich, Informationsheft, NÖ Landschaftsfonds, Austria.

Ministere DE L’Agriculture ET DE LA PECHe, 2006, Plan stratégique national de développement rural 2007-2013, Paris.

Mitchell G., MAY A., McDonald A., 1995, « PICABUE : a methodological framework for the development of indicators of sustainable development ", International Journal of Sustainable Development of World Ecology, vol. 2, pp. 104-123.

Mouillot D., LePetre A., 1999, « A comparison of species diversity estimators », Researches on Population Ecology, vol. 41, n², pp. 203-215.

NiemeiJer D., De Groot R. S., 2008, « A conceptual framework for selecting environmental indicator sets », Ecological Indicators, n ${ }^{\circ}$, pp. 14-25. 
Author-produced version of the article published in Revue d'Economie Régionale et Urbaine (RERU), 2010, vol. 3, p. 439 - 471 The original publication is available at http://www.cairn.info/resume.php?ID_ARTICLE=RERU_103_0439

doi : $10.3917 /$ reru. 103.0439

OCDE, 1999a, Indicateur environnementaux pour l'agriculture - Concepts et cadre d'analyse vol. 1, Edition OCDE, Paris.

OCDE, 1999b, Indicateur environnementaux pour l'agriculture - Questions clés et conception "Le séminaire de York" - vol. 2, Edition OCDE, Paris.

OCDE, 2001, Indicateur environnementaux pour l'agriculture - Méthodes et résultats, vol. 3, Edition OCDE, Paris.

OREADE-BRECHE, 2005, Evaluation des mesures agro-environnementales, Rapport final. Auzeville, 233 p.

OsSARD A., 2008, Évaluer et cartographier les risques d'impacts environnementaux, Campagnes et Environnement, hors série, pp. 6-7.

PAyraudeau S. et VAN DER Werf H. M.G., 2005, « Environmental impact assessment for a farming region : a review of methods ", Agriculture, Ecosystems and Environment, vol. 107, pp. 1-19.

PDRH, 2008, Programme de Développement Rural Hexagonal, Tome 2, version 3, Ministère de l’Agriculture et de la Pêche, Paris.

Perret B., 2008, L’évaluation des politiques publiques, La découverte, Coll. Repères, Paris.

Pingault N., Pleyber E., Champeaux C., Guichard L., OmOn B., « Produits phytosanitaires et protection intégrée des cultures : l'indicateur de fréquence de traitement », Notes et études socio-économiques, vol. 32, pp. 61-94.

PIORR H-P., 2003, « Environmental policy, agri-environmental indicators and landscape indicators », Agriculture, Ecosystems and Environment, 98, pp. 17-33.

Primdahl J., Peco B. , SchrameKC J., Andersend E., ONªteB J.J, 2003, « Environmental effects of agri-environmental schemes in Western Europe », Journal of Environmental Management, vol. 67, pp. 129-138.

Rosnoblet J., Girardin P., Weinzaepflen E., Bockstaller C., 2006, Analysis of 15 years of agriculture sustainability evaluation methods, In : Fotymâ, M, Kaminska, B. (Eds.), 9 th ESA Congress Warsaw, Poland, pp. 707-708.

RuBIN D., 1974, Estimating causal effect of treatment in randomized and non randomized studies, Journal of Educational Methods, $\mathrm{n}^{\circ}$ 66, pp. 688-701. 
Author-produced version of the article published in Revue d'Economie Régionale et Urbaine (RERU), 2010, vol. 3, p. 439 - 471 The original publication is available at http://www.cairn.info/resume.php?ID_ARTICLE=RERU_103_0439

doi : $10.3917 /$ reru. 103.0439

Schmitt B., Lofredi P., Berriet-Solliec M., LÉPICIER D., 2003, « Impact Evaluation of the EU Programme for Rural Development in Burgundy Correcting for Selection Bias », CESAER Working Papers, 2003/6, 29 p.

SCHOMAKER M., 1997, « Development of environmental indicators in UNEP », in : Land Quality Indicators and their Use in Sustainable Agriculture and Rural Development, January, FAO, Rome, pp. 35-36.

SolAGRO, 2000, DIAgnostic Liant Environnement et CTE - note explicative, Solagro, Toulouse.

Toulemonde J., 1997, « Faut-il libérer l'évaluation de ses liens de causalité ? Une réponse illustrée par les politiques structurelles européennes », Annales des Mines, pp. 76-88.

Udo de Haes H.A., Jolliet O., Finnveden G., Hauschild M., Krewitt W., Muller-Wenk R., 1999, « Best available practice regarding impact categories and catagory indicators in Life Cycle Impact Assessment - Background document for the second working group on Life », International Journal of Life Cycle Assessment, vol. 4, n ${ }^{\circ} 2$, pp. 66-74.

Van Caumenbergh N., Biala K., Bielders C., Brouckaert V., Franchois L., Garcia Cidad V., Hermy M., Mathiss E., Muys B., Reijnders J., Sauvenier X., Valckx J., Vanclooster M., Van Der Veken B., Wauters E., Peeters A., 2007, « SAFE-A hierarchical framework for assessing the sustainability of agricultural systems », Agriculture, Ecosystems and Environment, vol. 120, n² 2-4, pp. 229-242.

VAN Der Werf H.M.G., Zimmer C., 1998, « An indicator of pesticide environmental impact based on a fuzzy expert system », Chemosphere, vol. 36, n 10, pp. 2225-2249.

Vilain L., Girardin P., Mouchet C., Viaux P., Zahm F., 2008, La méthode IDEA Indicateurs de durabilité des exploitations agricoles, 3ème édition, Ed. Educagri, Dijon.

Zahm F., Chabe-Ferret S., Gassiat A., Subervie J., 2008a, « Proposition d'un cadre méthodologique pour évaluer les effets propres des mesures agro-environnementales européenne - Application au dispositif français », In : L'évaluation des politiques publiques en Europe - Cultures et futurs, Strasbourg, juillet 2008, 11 p.

Zahm F., Chabe-Ferret S., Gassiat A., Subervie J., 2008b, Assistance méthodologique à la préparation de l'évaluation ex-post du PDRN 2000-2006 en matière de soutien à l'agroenvironnement, Rapport final de synthèse, Cemagref, 174 p. 
Author-produced version of the article published in Revue d'Economie Régionale et Urbaine (RERU), 2010, vol. 3, p. 439 - 471 The original publication is available at http://www.cairn.info/resume.php?ID_ARTICLE=RERU_103_0439 doi : $10.3917 /$ reru.103.0439

Zalidis G., Tsiafouli M., TAKavakoglou V., Bilas G., Misopolinos N., 2004, «Selecting agri-environmental indicator to facilitate monitoring and assessment of EU agrienvironmental measures effectiveness», Journal of Environmental Management, vol. 70, $\mathrm{n}^{\circ}$ 4, pp. 315-321. 
Author-produced version of the article published in Revue d'Economie Régionale et Urbaine (RERU), 2010, vol. 3, p. 439 - 471 The original publication is available at http://www.cairn.info/resume.php?ID_ARTICLE=RERU_103_0439 doi : $10.3917 /$ reru. 103.0439

Annexe 1 : Les enjeux environnementaux au regard des pratiques agricoles en lien avec les questions évaluatives de la Commission européenne

\begin{tabular}{|c|c|c|c|c|}
\hline \multicolumn{2}{|c|}{ Enjeux } & Code & Pratiques agricoles & $\begin{array}{l}\text { Questions évaluatives } \\
\text { du cadre commun }\end{array}$ \\
\hline \multirow{2}{*}{\multicolumn{2}{|c|}{ के }} & SOL1 & $\begin{array}{l}\text { maîtriser l'érosion du sol (réduire la surface de sol nu, } \\
\text { aménager l'espace agricole) }\end{array}$ & \multirow{2}{*}{$\begin{array}{l}\text { Dans quelle mesure les ressources } \\
\text { naturelles ont-elles été protégées en } \\
\text { terme de qualité du sol sous } \\
\text { l’influence des MAE? }\end{array}$} \\
\hline & & SOL2 & $\begin{array}{l}\text { maintenir la qualité ou la fertilité des sols (notamment } \\
\text { prévenir ou réduire les apports d'intrants chimiques ou } \\
\text { organiques) }\end{array}$ & \\
\hline \multirow{6}{*}{ 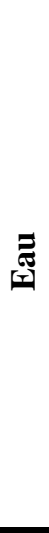 } & \multirow{2}{*}{ 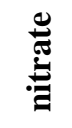 } & EAUn1 & réduire les apports (nitrate) & \multirow{4}{*}{$\begin{array}{l}\text { Dans quelle mesure les ressources } \\
\text { naturelles ont-elles été protégées en } \\
\text { terme de qualité des eaux sous } \\
\text { l'influence des MAE? }\end{array}$} \\
\hline & & EAUn2 & réduire les transferts (nitrate) & \\
\hline & \multirow{2}{*}{ 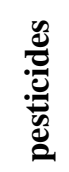 } & EAUp1 & réduire les apports (pesticides) & \\
\hline & & EAUp2 & réduire les transferts (pesticides) & \\
\hline & \multirow{2}{*}{. } & EAUq1 & réduire les surfaces en cultures irriguées & \multirow{2}{*}{$\begin{array}{l}\text { Dans quelle mesure les ressources } \\
\text { naturelles ont-elles été protégées en } \\
\text { terme de quantité de ressources } \\
\text { hydriques sous l'influence des } \\
\text { MAE? }\end{array}$} \\
\hline & & EAUq2 & réduire le niveau d'irrigation à l'hectare & \\
\hline \multirow{7}{*}{ : } & \multirow{4}{*}{ 莽 } & BIOc1 & réduire les intrants & \multirow{4}{*}{$\begin{array}{l}\text { Dans quelle mesure la biodiversité } \\
\text { a-t-elle été préservée ou améliorée, } \\
\text { grâce aux MAE, par la protection } \\
\text { faune et flore sur les terres } \\
\text { agricoles? }\end{array}$} \\
\hline & & BIOc2 & rotation des cultures & \\
\hline & & BIOc3 & végétation en période critique & \\
\hline & & BIOc4 & gestion écologique & \\
\hline & \multirow{3}{*}{ 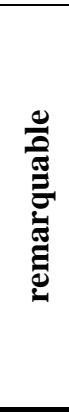 } & BIOr1 & $\begin{array}{l}\text { conservation des espèces faunistiques et floristiques } \\
\text { remarquables }\end{array}$ & \multirow{2}{*}{$\begin{array}{l}\text { Dans quelle mesure la biodiversité } \\
\text { a-t-elle été préservée ou améliorée, } \\
\text { grâce aux MAE, par la } \\
\text { conservation des habitats HVN et } \\
\text { la protection des IAE? }\end{array}$} \\
\hline & & BIOr2 & conservation des habitats & \\
\hline & & BIOr3 & $\begin{array}{l}\text { conservation des races domestiques animales et } \\
\text { végétales menacées }\end{array}$ & $\begin{array}{l}\text { Dans quelle mesure la biodiversité } \\
\text { a-t-elle été préservée ou améliorée, } \\
\text { grâce aux MAE, par la } \\
\text { préservation des races et variétés } \\
\text { menacées? }\end{array}$ \\
\hline \multirow{3}{*}{\multicolumn{2}{|c|}{ 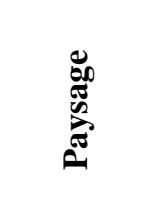 }} & PAY1 & diversification & \multirow{3}{*}{$\begin{array}{l}\text { Dans quelle mesure les paysages } \\
\text { ont-ils été préservés ou améliorés } \\
\text { par les MAE? }\end{array}$} \\
\hline & & PAY2 & identité territoriale & \\
\hline & & PAY3 & identité culturelle & \\
\hline
\end{tabular}

HVN : Haute valeur naturelle IAE : Infrastructure agro-écologique 
Author-produced version of the article published in Revue d'Economie Régionale et Urbaine (RERU), 2010, vol. 3, p. 439 - 471 The original publication is available at http://www.cairn.info/resume.php?ID_ARTICLE=RERU_103_0439 doi : $10.3917 /$ reru.103.0439

\section{Annexe 2 : Organisation de la BIAE pour chaque indicateur}

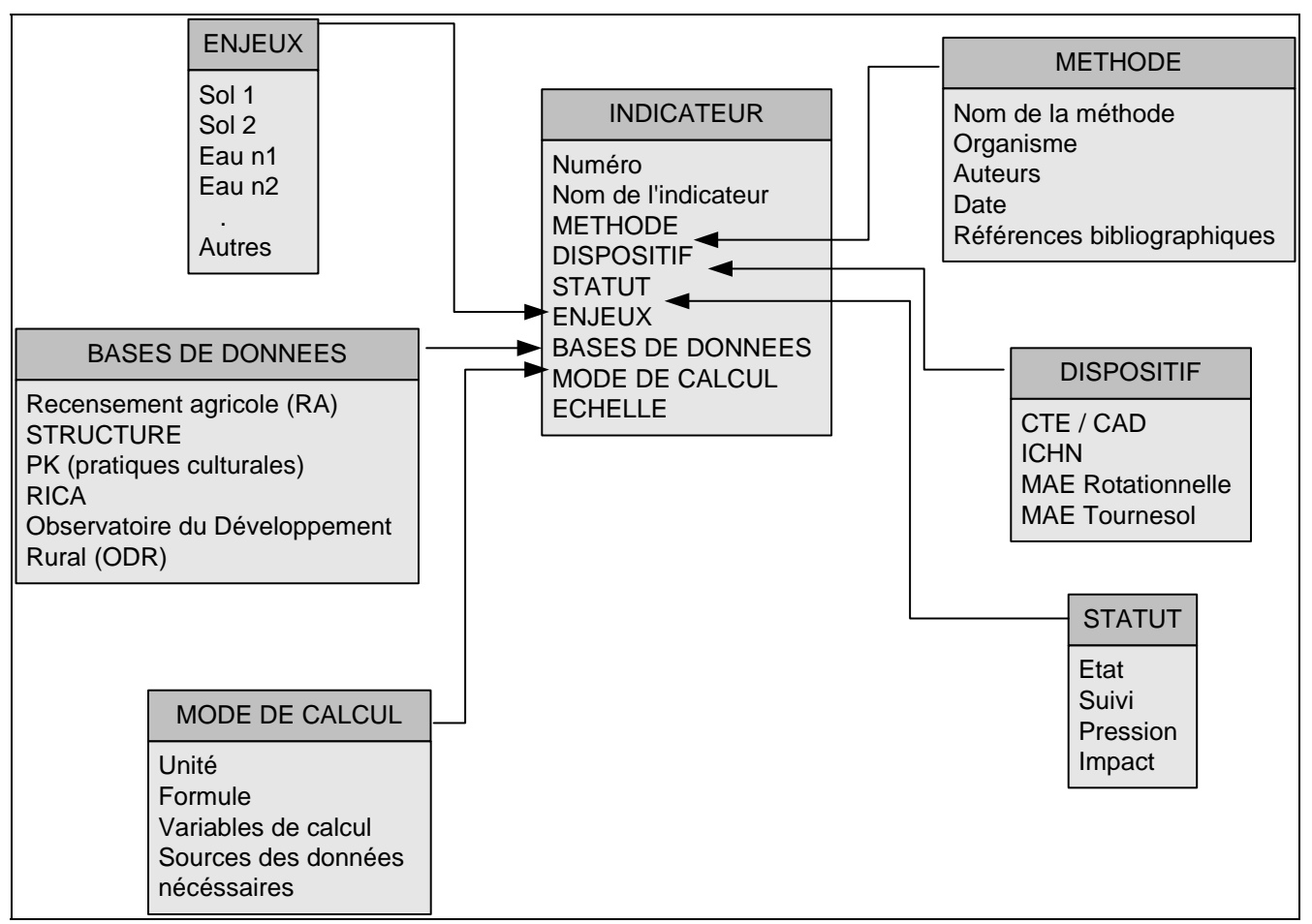


Author-produced version of the article published in Revue d'Economie Régionale et Urbaine (RERU), 2010, vol. 3, p. 439 - 471 The original publication is available at http://www.cairn.info/resume.php?ID_ARTICLE=RERU_103_0439 doi : 10.3917/reru.103.0439

\section{Annexe 3 : Liste des 40 indicateurs calculables}

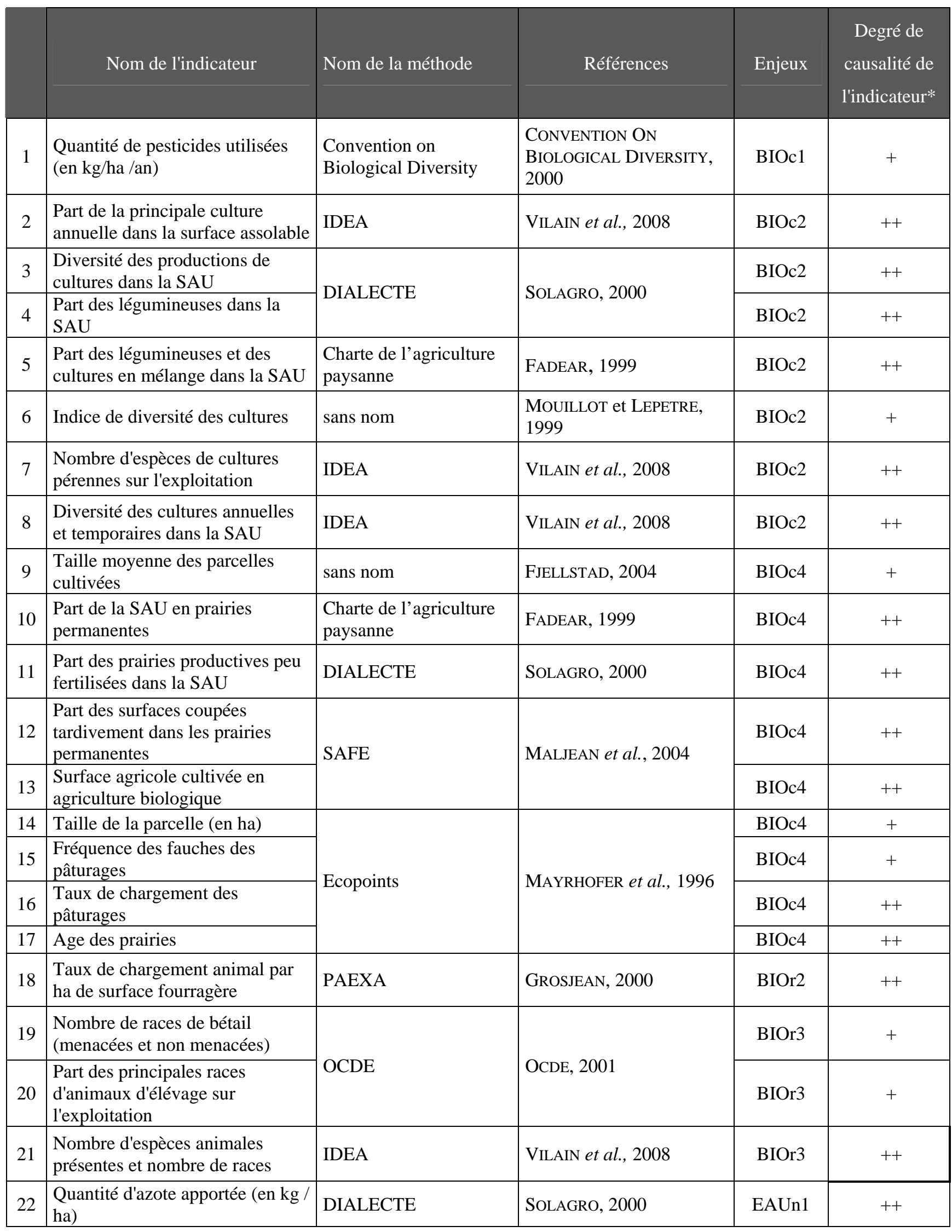


Author-produced version of the article published in Revue d'Economie Régionale et Urbaine (RERU), 2010, vol. 3, p. 439 - 471 The original publication is available at http://www.cairn.info/resume.php?ID_ARTICLE=RERU_103_0439 doi : 10.3917/reru.103.0439

\begin{tabular}{|c|c|c|c|c|c|}
\hline & Nom de l'indicateur & Nom de la méthode & Références & Enjeux & $\begin{array}{c}\text { Degré de } \\
\text { causalité de } \\
\text { l'indicateur* }\end{array}$ \\
\hline 23 & $\begin{array}{l}\text { Part de la SAU amendée avec de } \\
\text { la matière organique }\end{array}$ & PAEXA & GROSJEAN, 2000 & EAUn1 & ++ \\
\hline 24 & Surplus d'azote au sol & IRENA & $\begin{array}{l}\text { EUROPEAN ENVIRONMENT } \\
\text { AGENCY, } 2005\end{array}$ & EAUn2 & ++ \\
\hline 25 & Bilan azoté corpen & CORPEN & CORPEN, 1988 & EAUn2 & ++ \\
\hline 26 & Bilan apparent de l'azote & IDEA & VILAIN et al., 2008 & EAUn2 & +++ \\
\hline 27 & $\begin{array}{l}\text { Part de la SAU avec une } \\
\text { couverture du sol en hiver }\end{array}$ & DIALECTE & SOLAGRO, 2000 & EAUn2 & ++ \\
\hline 28 & $\begin{array}{l}\text { Couverture végétale du sol } \\
\text { pendant la période de lessivage }\end{array}$ & SAFE & MALJEAN et al., 2004 & EAUn2 & + \\
\hline 29 & $\begin{array}{l}\text { Surface de culture de pièges à } \\
\text { nitrate }\end{array}$ & sans & CORPEN, 2006 & EAUn2 & ++ \\
\hline 30 & $\begin{array}{l}\text { Surfaces cultivées non traitées } \\
\text { avec des pesticides d'origine } \\
\text { chimique }\end{array}$ & $\begin{array}{l}\text { Environmental } \\
\text { indicators for } \\
\text { agriculture }\end{array}$ & OCDE, 2001 & EAUp1 & ++ \\
\hline 31 & $\begin{array}{l}\text { Indice de fréquence de traitement } \\
\text { (pesticides) }\end{array}$ & IFT & GRAVESEN, 2003 & EAUp1 & + \\
\hline 32 & $\begin{array}{l}\begin{array}{l}\text { Surface développée traitée en } \\
\text { pesticides }\end{array} \\
\end{array}$ & IDEA & VILAIN et al., 2008 & EAUp1 & ++ \\
\hline 33 & $\begin{array}{l}\text { Absence ou faible utilisation de } \\
\text { pesticides }\end{array}$ & DIALECTE & SOLAGRO, 2000 & EAUp1 & ++ \\
\hline 34 & $\begin{array}{l}\text { Linéaires de bandes enherbées } \\
\text { (en m.) }\end{array}$ & sans nom & CORPEN, 2003 & EAUp2 & + \\
\hline 35 & $\begin{array}{l}\text { Part des surfaces irriguées dans } \\
\text { la SAU }\end{array}$ & \multirow{2}{*}{ Little Green Databook } & \multirow{2}{*}{$\begin{array}{l}\text { INSTITUT INTERNATIONAL } \\
\text { DU DEVELOPPEMENT } \\
\text { DURABLE, } 2002\end{array}$} & EAUq1 & + \\
\hline 36 & $\begin{array}{l}\text { Part des terres irriguées dans la } \\
\text { SAU }\end{array}$ & & & EAUq1 & + \\
\hline 37 & $\begin{array}{l}\text { Part de la SAU irriguée et type } \\
\text { de matériel }\end{array}$ & IDEA & VILAIN et al., 2008 & EAUq1 & ++ \\
\hline 38 & $\begin{array}{l}\text { Volume d'eau apportée pour } \\
\text { l'irrigation (m3 /ha /an) }\end{array}$ & \multirow{2}{*}{ DAEG } & \multirow{2}{*}{ OSSARD, 2008} & EAUq2 & +++ \\
\hline 39 & $\begin{array}{l}\text { Volume d'eau consommé sur } \\
\text { l'exploitation (en m3) }\end{array}$ & & & EAUq2 & +++ \\
\hline 40 & $\begin{array}{l}\text { Volume d'eau apporté pour } \\
\text { l'irrigation (m3/ha /an) }\end{array}$ & IRENA & $\begin{array}{l}\text { EUROPEAN ENVIRONMENT } \\
\text { AGENCY, } 2005\end{array}$ & EAUq2 & ++ \\
\hline
\end{tabular}

légende* : + degré faible, ++ degré moyen, +++ degré élevé SAU: Surface agricole Utile 